\title{
New integrated chassis control systems for vehicle handling performance enhancement
}

\author{
Ahmed Elmarakbi • Chandrasekaran Rengaraj • \\ Alan Wheately • Mustafa Elkady
}

Received: 11 June 2013 / Revised: 19 August 2013 / Accepted: 23 August 2013 / Published online: 6 September 2013

(C) Springer-Verlag Berlin Heidelberg 2013

\begin{abstract}
This paper investigates the principle of integration of vehicle dynamics control systems by proposing a new control architecture to integrate the following four major functional domains of a vehicle; braking, steering, suspension and driveline. The active control systems include brakebased electronic stability control, active front steering, normal suspension force control and variable torque distribution. Based on the analysis of these four standalone controllers, a novel rule based integration strategy is proposed to improve the vehicle handling. A nonlinear vehicle handling model is developed for this study in Matlab/Simulink. This model contains a sprung mass of six degrees of freedom that includes, longitudinal, lateral, yaw, roll, pitch and bounce and un-sprung masses with wheels at each corner of the vehicle. The simulation results show that this integration strategy enhances the vehicle handling stability in terms of reduction in vehicle yaw rate and side-slip angle that would not be attained in standalone manner.
\end{abstract}

Keywords Integrated control system - Vehicle dynamics · Performance $\cdot$ Handling $\cdot$ Numerical simulations

\section{List of symbols}

$\begin{array}{ll}a & \text { Longitudinal distance of CG from front axle } \\ a_{x} & \text { Longitudinal acceleration at CG } \\ a_{y} & \text { Lateral acceleration at CG } \\ b & \text { Longitudinal distance of CG from rear axle } \\ c & \text { Tyre cornering stiffness }\end{array}$

A. Elmarakbi ( $₫)$. C. Rengaraj · A. Wheately · M. Elkady

Department of Computing, Engineering and Technology, Faculty of Applied Sciences, University of Sunderland, Sunderland SR6 0DD, UK e-mail: ahmed.elmarakbi@sunderland.ac.uk

\begin{tabular}{|c|c|}
\hline$B, C, D, E$ & Pacejka tyre parameters \\
\hline$F_{n}$ & Tyre normal force \\
\hline$F_{s i j}$ & Suspension force \\
\hline$F_{S}$ & Suspension vertical force \\
\hline$F_{t}$ & Tyre vertical force \\
\hline$F_{x i j}$ & Longitudinal tyre force \\
\hline$F_{x s s}$ & Longitudinal steady state tyre force \\
\hline$F_{y i j}$ & Lateral tyre force \\
\hline$F_{y s s}$ & Lateral steady state tyre force \\
\hline$g$ & Gravitational constant \\
\hline$h_{c g}$ & Height of centre of gravity from ground \\
\hline$i$ & Stands for front/rear $(f, r)$ \\
\hline$I_{w}$ & Wheel rotational inertia \\
\hline$I_{x x}$ & $\begin{array}{l}\text { Sprung mass roll moment of inertia at } \mathrm{CG} \text { of } \\
\text { vehicle }\end{array}$ \\
\hline$I_{y y}$ & $\begin{array}{l}\text { Sprung mass pitch moment of inertia at CG of } \\
\text { vehicle }\end{array}$ \\
\hline$I_{z z}$ & $\begin{array}{l}\text { Sprung mass yaw moment of inertia at } \mathrm{CG} \text { of } \\
\text { vehicle }\end{array}$ \\
\hline$j$ & Stands for left/right $(l, r)$ \\
\hline$K_{b}$ & Brake gain of the brake system \\
\hline$K_{u s}$ & Under steering gradient \\
\hline$L$ & Wheel base \\
\hline$l_{f}$ & Distance of vehicle CG from front axle \\
\hline$L_{F x}$ & Longitudinal tyre force lag \\
\hline$L_{F y}$ & Lateral tyre force lag \\
\hline$l_{r}$ & Distance of vehicle CG from rear axle \\
\hline$M_{S}$ & Sprung-mass of vehicle \\
\hline$m_{u s i j}$ & Vehicle unsprung mass \\
\hline$M_{v}$ & Mass of total vehicle \\
\hline$M_{z c}$ & Desired corrective yaw moment \\
\hline$P_{b i j}$ & Brake pressure \\
\hline$R_{w}$ & Dynamic tyre radius \\
\hline$t$ & Track width of vehicle \\
\hline$T$ & Drive torque on wheels \\
\hline
\end{tabular}




$\begin{array}{ll}t_{f} & \text { Vehicle front track width } \\ t_{r} & \text { Vehicle rear track width } \\ V_{c h} & \text { Characteristic speed } \\ V_{x} & \text { Sprung mass longitudinal velocity at CG } \\ \dot{V}_{x} & \text { Derivative of longitudinal velocity } \\ V_{y} & \text { Sprung mass lateral velocity at CG } \\ \dot{V}_{y} & \text { Derivative of lateral velocity } \\ V_{z} & \text { Sprung mass vertical velocity at CG } \\ \dot{V}_{z} & \text { Derivative of vertical velocity } \\ Z_{s} & \text { Sprung mass vertical displacement } \\ \dot{Z}_{s} & \text { Sprung mass vertical velocity } \\ Z_{u s} & \text { Unsprung mass vertical displacement } \\ \dot{Z}_{u s} & \text { Unsprung mass vertical velocity } \\ \alpha & \text { Tyre lateral slip angle } \\ \beta & \text { Side-slip angle } \\ \delta & \text { Steering angle at the front wheels } \\ \theta & \text { Vehicle pitch angle at CG } \\ \dot{\theta} & \text { Vehicle pitch rate at CG } \\ \ddot{\theta} & \text { Derivative of vehicle pitch velocity } \\ \lambda & \text { Tyre longitudinal slip ratio } \\ \mu & \text { Coefficient of friction } \\ \phi & \text { Vehicle roll angle at CG } \\ \dot{\phi} & \text { Vehicle roll rate at CG } \\ \ddot{\phi} & \text { Derivative of vehicle roll velocity } \\ \psi & \text { Vehicle yaw angle at CG } \\ \dot{\psi} & \text { Vehicle yaw rate at CG } \\ \dot{\psi} & \text { Reference yaw rate } \\ \ddot{\psi} & \text { Derivative of vehicle yaw velocity } \\ \omega & \text { Angular wheel velocity } \\ & \end{array}$

\section{Introduction}

Today's modern vehicles include more than 40 actively controlled systems that play a major role in vehicle directional stability, ride comfort and safety. Several significant control systems, such as anti-lock braking systems (ABS) [13], active suspension systems [4], traction control systems, Active yaw control and so forth, are being popularly used in automobile industries. Many theories and design methods for active yaw control, anti-lock braking systems and active suspension systems have been proposed individually by several literatures for decades. Various researchers have considered a slip-ratio control of anti-lock braking systems in the use of sliding mode control schemes [3]. As to the design of active suspensions, the improvement of ride comfort is the major objective to be emphasized. A novel nonlinear back-stepping design $[4,5]$ has been developed for a quarter-car active suspension system which aims to improve the trade-off between the ride quality of passenger comfort and the utilization of suspension travel. For further improving vehicle handling and stability, yaw moment control has been studied and developed by controlling braking force distribution of four wheels as a strategy for driver support systems.

At present these systems generally work independently but it is widely accepted that integration of these standalone systems will lead to improved vehicle dynamic performance [6]. Additional benefits include cost and weight reductions and reduced sensor requirements. Both the automotive industry and the end users will benefit directly from this research. However, successful integration of such control systems is still largely in the research phase. Previous studies have identified that these systems were originally developed independently to perform specific tasks and some systems do co-exist, Junjie et al. [7], Karbalei et al. [8], and Kazuya and Peng [9]. Researchers have succeeded in the successful integration of several systems, March and Shim [10]; however, potential conflicts are still a problem. Complete integration of many sub-systems is still a real technical challenge.

The overall aim of this paper is to develop new control strategies/algorithms to enable a successful integration of a subset of vehicle control systems. However, this paper focuses primarily on the methods of improving vehicle stability and emergency handling through the integration of four specific vehicle control systems: active front steering (AFS), normal suspension force control (NFC), brake-based electronic stability control (ESC) and driveline based variable torque distribution (VTD) system.

There are many ways to compare the performance improvement obtained by an integrated chassis controller against its standalone counterpart. Few of the techniques include comparing the reduction in energy consumption, reduced cost, less/modular parts, improvement in performance variable etc. In this paper, the improved performance objectives established from using the integrated chassis control approach are defined as a reduction in yaw rate and vehicle side-slip angle that lead to better handling capabilities.

The rest of the paper is organized as follows. In the vehicle modelling section, a detailed passive vehicle dynamics model with nonlinear tyres suitable for combined slip and transient conditions is developed in Matlab/Simulink environment along with the dynamics of steering, braking, suspension and driveline systems. In the standalone control systems section, the development of standalone control system models of AFS, active suspension, a brake based ESC and a VTD system are discussed. Various possible integrated control strategies amongst those systems in consideration are analyzed and investigated in the integrated control system section. This section also explains a new integrated chassis control (ICC) strategy developed from the results of the analysis and implemented in MATLAB/Simulink. Finally the conclusions based on the new ICC strategy are illustrated. 


\section{Vehicle modelling}

The body of the vehicle model used in this paper is assumed to be rigid and has six degrees of freedom (DoF, three translational and three rotational). The vehicle axis co-ordinate system used is assumed to be fixed at the CG of the vehicle body. The vehicle's equations of motion are derived with reference to both the vehicle and inertial co-ordinate systems. It is assumed that a suspension unit is attached at each corner of the vehicle with linear spring and damper elements. The dynamics of un-sprung mass and tyre at each corner are also included in this model. As full vehicle modelling is not a simple task and it involves many subsystems and coupled nonlinear system dynamics, certain modelling assumptions are made and the same are explained here.

- The self-aligning moments of the tyre is neglected as they do not disturb the vehicle dynamics by bringing back the steering wheel to the initial position.

- The kinematics effects due to suspension geometry are neglected. So the suspensions only provide vertical force to the chassis.

- The gyroscopic effects of the sprung mass are neglected. The only external forces acting on the vehicle is assumed to the longitudinal, lateral and vertical forces generated by the tyres.

- The tyre cambering is considered in tyre modelling.

- The vehicle chassis plane is considered parallel to the ground.

- The aero dynamical and wheel friction effects are neglected as in this work study of those effects is not of great interest.

where $Z_{s}$ is the sprung mass vertical displacement, $\phi$ and $\theta$ are the roll and pitch angle of the chassis respectively, $l_{f}, l_{r}, t_{f}, t_{r}$ stands for the vehicle geometry. where $l_{f}$ and $l_{r}$ are the distances of vehicle CG from front and rear axle, respectively, and $t_{f}$ and $t_{r}$ are the vehicle front and rear track width, respectively.

The full vehicle model is defined by the following nonlinear dynamical equations.

$\dot{V}_{x}=\left\{\frac{\sum F_{x i j}}{M_{v}}\right\}-V_{y} \dot{\psi}+V_{z} \dot{\theta}$

where

$$
\begin{gathered}
\sum F_{x i j}=\left(F_{x f r}+F_{x f l}\right) \cos (\delta)+F_{x r r} \\
+F_{x r l}-\left(F_{y f r}+F_{y f l}\right) \sin (\delta) \\
\dot{V}_{y}=\left\{\frac{\sum F_{y i j}}{M_{v}}\right\}+V_{x} \dot{\psi}-V_{z} \dot{\phi},
\end{gathered}
$$

where

$$
\begin{aligned}
& \sum F_{y i j}=\left(F_{y f r}+F_{y f l}\right) \cos (\delta)+F_{y r r} \\
& +F_{y r l}+\left(F_{x f r}+F_{x f l}\right) \sin (\delta) \\
& \dot{Z}_{s}=\left\{\frac{\sum F_{s i j}}{M_{s}}\right\}+V_{x} \dot{\theta}-V_{y} \dot{\phi},
\end{aligned}
$$

where

$$
\begin{aligned}
& \sum F_{s i j}=F_{s f l}+F_{s f r}+F_{s r l}+F_{s r r} \\
& \ddot{Z}_{u s i j}=\frac{\left(-F_{s z i j}+F_{t z i j}\right)}{M_{u s i j}}
\end{aligned}
$$

$\ddot{\theta}=\frac{\left\{\left(F_{s r l}+F_{s r r}\right) l_{r}-\left(F_{s f l}+F_{s f r}\right) l_{f}-M_{s} h_{c g} a_{x}+\left(I_{z z}-I_{x x}\right) \dot{\psi} \dot{\phi}\right\}}{I_{y y}}$

$\ddot{\phi}=\frac{\left\{\left(F_{s f l}+F_{s r l}\right) t_{f}-\left(F_{s f r}+F_{s r r}\right) t_{f}-M_{s} h_{c g} a_{y}+\left(I_{y y}-I_{z z}\right) \dot{\psi} \dot{\theta}\right\}}{I_{x x}}$

- The effects due to the toe-in and toe-out of the tyres are neglected.

A schematic view of the nonlinear vehicle model used is shown in Fig. 1.

The kinematics equations are mainly due to the vehicle geometry. Each corner of the vehicle is identified with $i, j$ index where $i=\{f, r\}$ stands for front/rear and $j=\{l, r\}$ stands for left/right. The displacements of the sprung mass on chassis corners are described by

$$
\begin{aligned}
& Z_{s f j}=Z_{s}-l_{f} \sin (\theta) \pm t_{f} \sin (\phi) \\
& Z_{s r j}=Z_{s}+l_{f} \sin (\theta) \pm t_{r} \sin (\phi),
\end{aligned}
$$

$$
\ddot{\psi}=\frac{\left\{\sum F_{m z}+\left(I_{x x}-I_{y y}\right) \dot{\phi} \dot{\theta}\right\}}{I_{z z}},
$$

where

$$
\begin{aligned}
\sum F_{m z}= & \sum F_{y f j} \cos (\delta) l_{f}-\sum F_{y r j} l_{r} \\
& +\sum F_{x f j} \cos (\delta) t_{f}-\sum F_{x r j} t_{r} .
\end{aligned}
$$

The forces are given by the following equations, Tyres [6]:

$$
\left\{\begin{array}{l}
F_{t x i j}=F_{t x}\left(\mu_{i j}, \lambda_{i j}, F_{n i j}\right) \\
F_{t y i j}=F_{t y}\left(\mu_{i j}, \alpha_{i j}, F_{n i j}\right) \\
F_{t z i j}=F_{t z}\left(Z_{u s}-Z_{r}\right)
\end{array}\right.
$$


Fig. 1 Schematic of nonlinear vehicle model

Fig. 2 Full vehicle model synopsis
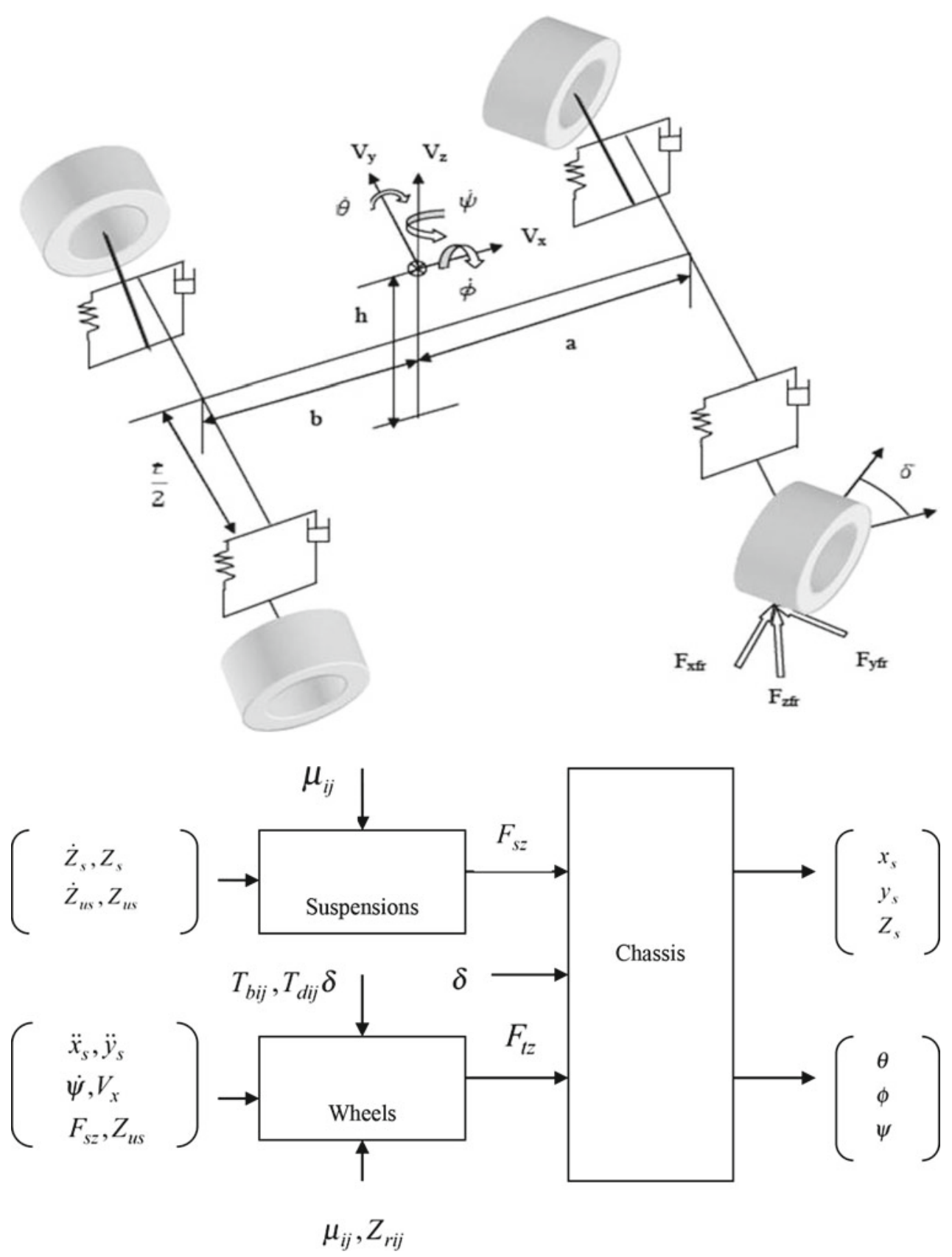

Suspensions:

$F_{s z i j}=F_{s z}\left(Z_{s i j}-Z_{u s i j}\right)$.

The normal force on each tyre is calculated based on the following equation

$F_{n z i j}=F_{n s_{-} i j}+F_{t z i j}$,

where $F_{n s_{-} i j}$ is the static load acting on the $i j$ th tyre.

A synopsis of data flow takes place amongst various vehicle subsystems in this model is given in Fig. 2. The vehicle model is divided into sub-models that describe the wheel, brake, suspension and steering dynamics.
The dynamics of the tyre-road interaction are dependent on the lateral and longitudinal tyre slips. The lateral tyre slip angles for each wheel can be calculated as follows:

$\alpha_{i}=\tan ^{-1}\left(\frac{V_{y}+a \dot{\psi}}{V_{x} \mp \frac{t}{2} \dot{\psi}}\right)-\delta ; \quad \alpha_{j}=\tan ^{-1}\left(\frac{V_{y}-b \dot{\psi}}{V_{x} \mp \frac{t}{2} \dot{\psi}}\right)$.

The component of the vehicle velocity of the wheel center that is parallel to the wheel vertical plane is given as 
$V_{i}=\cos \left(\alpha_{i}\right) \sqrt{\left(V_{x} \mp \frac{t}{2} \dot{\psi}\right)^{2}+\left(V_{y}+a \dot{\psi}\right)^{2}}$

$V_{j}=\cos \left(\alpha_{j}\right) \sqrt{\left(V_{x} \mp \frac{t}{2} \dot{\psi}\right)^{2}+\left(V_{y}-b \dot{\psi}\right)^{2}}$.

The longitudinal wheel slip is defined as

$\lambda_{i j}=\left\{\begin{array}{ll}\frac{R_{w} \omega_{i}-V_{x i}}{R_{w} \omega_{i}} & (\text { driving }) \\ \frac{V_{x i}-R_{w} \omega_{i}}{V_{x i}} & \text { (braking) }\end{array}\right.$.

Capturing the tyre behavior is probably the most difficult and important problem to tackle while building a vehicle model as realistically as possible. In the past, a lot of different models have been created to solve this problem. The most realistic models are the most complicated but probably they are not useful in every kind of research. On account of our objectives, a too simple model is not applicable because it can provide correct results only if the slip angles are very small, but it cannot represent for example the forces the tyres transfer during an emergency handling maneuver. For this reason a semi-empirical model usually called "Magic formula" suggested by Pacejka [11] is chosen. For simplification, the camber has been set to zero in the current vehicle model. The general equation of the tyre model is as follows:

$y(x)=D \sin \left[C \tan ^{-1}\left(B x-E\left(B x-\tan ^{-1}(B x)\right)\right)\right]$,

where $y(x)$ is $F_{x}$ and $F_{y}$, respectively, if $x$ is $\lambda$ or $\alpha$.

The tyre forces generated using the above equations in longitudinal and lateral directions are a function of pure slips in their respective directions. But in reality, these tyre forces are generated as a function of combined slip that exists during typical combined braking and cornering situations such as braking before entering a corner and accelerating before exiting it. Weighting functions $G$ as described in [12] are introduced which when multiplied with the original pure slip functions produce the interactive effects of longitudinal slip on $F_{y}$ and lateral slip on $F_{x}$. The cosine version of the magic formula is used to represent the hill shaped weighting function $G$ :

$G=D \cos \left[C \tan ^{-1}(B x)\right]$.

The combined side force is described by the following formulae:

$F_{y}=G_{y k} \cdot F_{y o}+S_{V y k}$,

where $S_{V y k}$, the effect due to ply-steer is assumed in this paper to be zero to reduce the complexity. The function $G_{y k}$ is used as described in [13]. The combined side force is described by the following formulae:
$F_{x}=G_{x \alpha} \cdot F_{x o}$,

where $G_{y k}$ and $G_{x \alpha}$ are described as follows

$G_{y k}=\frac{\cos \left[C_{y k} \tan ^{-1}\left(B_{y k} k_{S}\right)\right]}{\cos \left[C_{y k} \tan ^{-1}\left(B_{y k} S_{H y k}\right)\right]} \quad$ and

$G_{y k}=\frac{\cos \left[C_{x \alpha} \tan ^{-1}\left(B_{x \alpha}\left(\alpha+S_{H x \alpha}\right)\right]\right.}{\cos \left[C_{x \alpha} \tan ^{-1}\left(B_{x \alpha} S_{H x \alpha}\right)\right]}$.

A detailed description of these weighing functions can be found in [14]. Figure 3 shows the longitudinal and lateral tyre forces in combined braking and cornering conditions used in this paper obtained using the above mentioned equations. The effect of tyre force lag [13] is also taken into account according to the following equations.

$\left.\begin{array}{l}\frac{L_{F x}}{V_{x}} \frac{d\left(F_{x}\right)}{d t}+F_{x}=F_{x s s} \\ \frac{L_{F y}}{V_{y}} \frac{d\left(F_{y}\right)}{d t}+F_{y}=F_{y s s}\end{array}\right\}$.

The equation of motion for each wheel in the wheel dynamics model is defined as

$I_{w} \dot{\omega}_{i j}=T_{i j}-F_{x i j} R_{w}$.

The steering system modelled in this work has a hydraulic power steering mechanism. The input for the steering system is the angle of the steering wheel and steering column, while the output is the position of the rack, which determines the angle of the front wheels. There is a mechanical connection between the rack and the steering column with a pinion gear, which converts the rotational motion of the steering column to translational motion of the rack to turn the wheels.

The power assistance is provided by a hydraulic piston attached to the rack. A torsion valve determines which side of the piston receives pressurized hydraulic fluid. This torsion valve is attached to the steering column. The difference between the angular position of the steering wheel and the angular position of the pinion determines the fractional opening of the torsion valve. If the angular difference is positive, the pressure is applied to one side of the piston, and if the angular difference is negative, the pressure is applied to the other side of the piston. The power assistance continues until the difference between the steering wheel position and pinion position is approximately zero. The steering power assist curve is shown in Fig. 4.

The hydraulic brake system considered in this study is built upon a standard braking model. The standard passive brake system considered for this study consists of a mechanical brake pedal, a servo brake booster, a master cylinder, a hydraulic brake caliper and the friction pad. The brake mechanics considered here are explained as follows. The mechanical brake input is amplified by the servo booster. This is further amplified and converted to a hydraulic pressure called line pressure/supply pressure, which is fed through the 
Fig. 3 Tyre forces in combined slip-friction ellipse

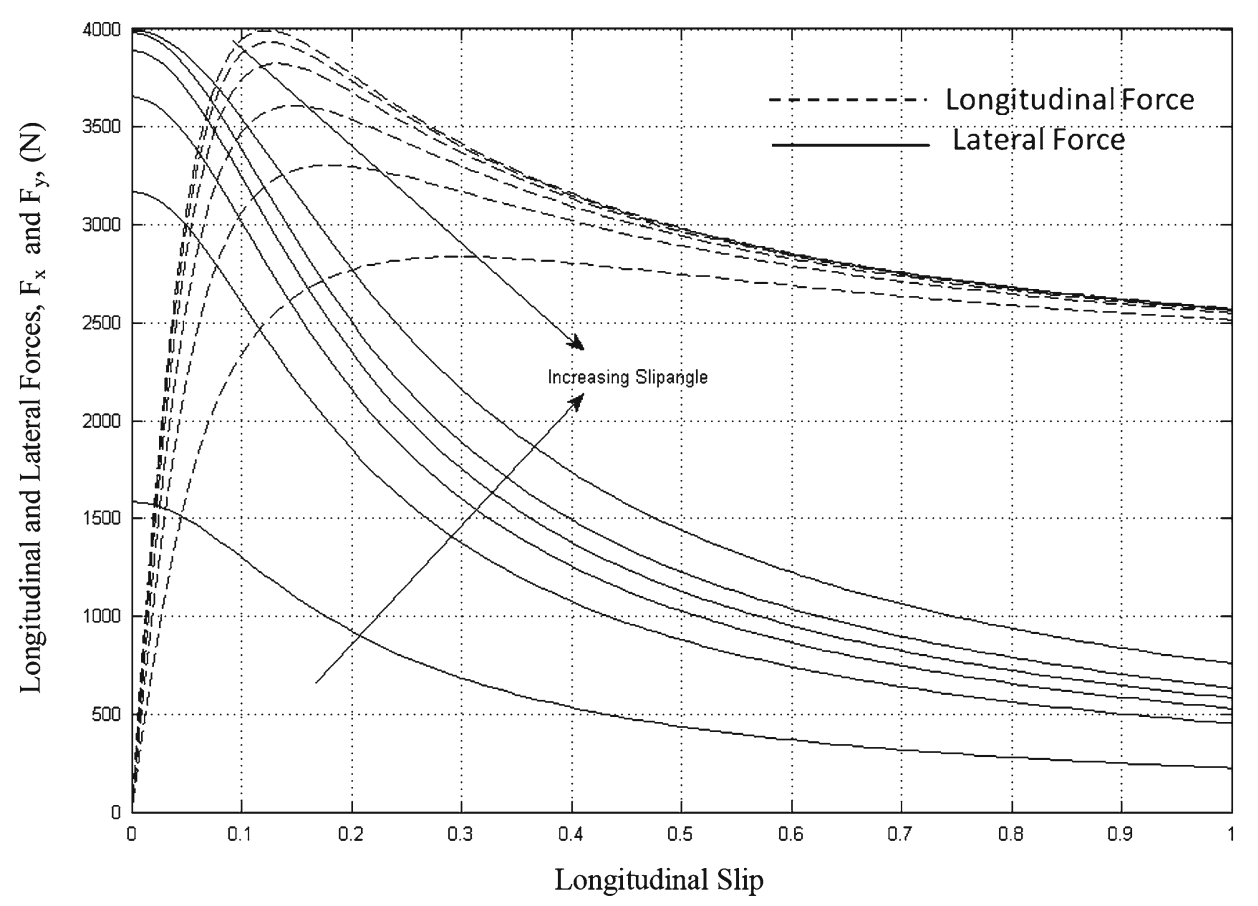

Combined Longitudinal and Lateral tyre force vs. slip ratio

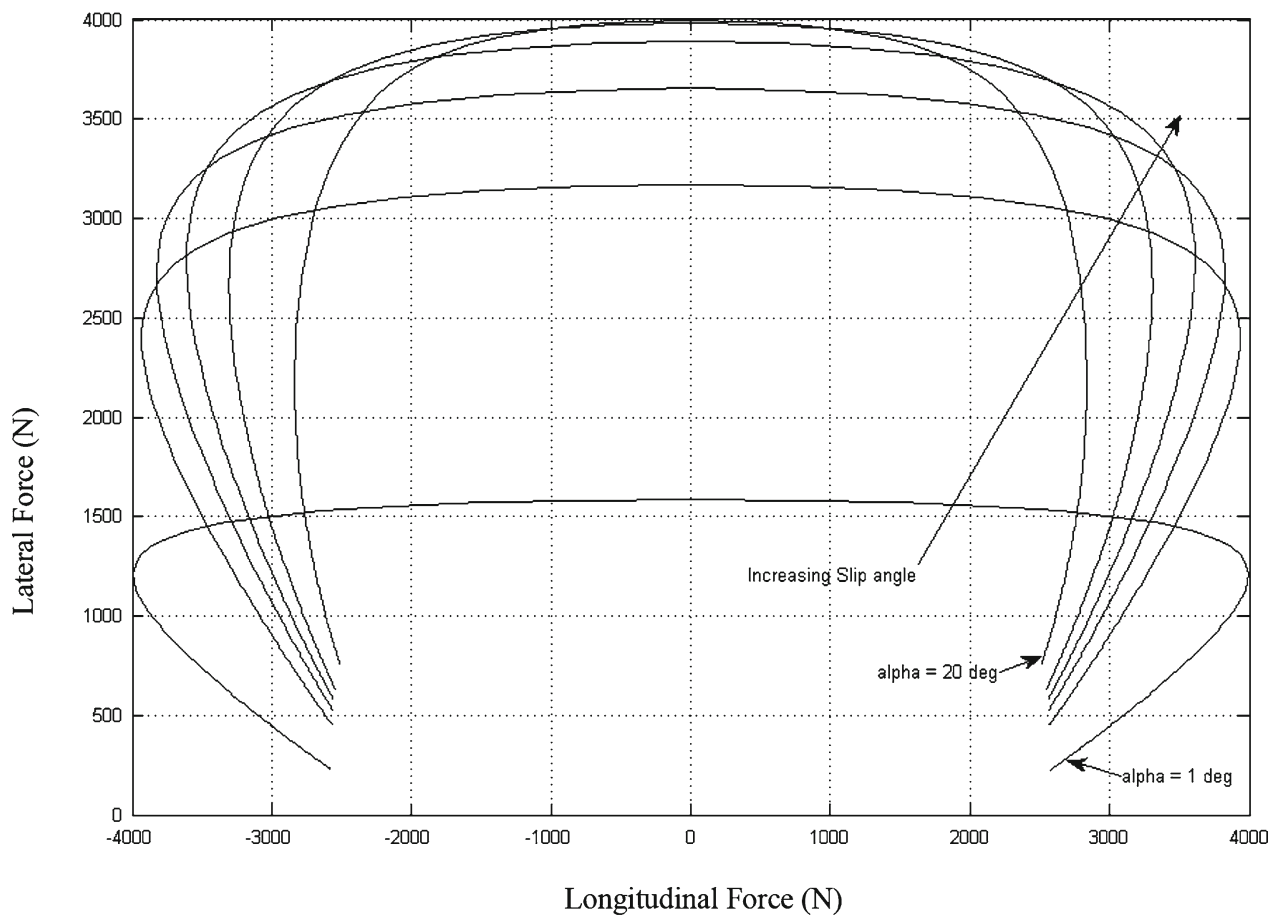

Tyre forces during combined braking and cornering brake lines. The line pressure is further amplified and converted to mechanical actuation at the brake calipers. This force moves the friction pads against the rotating wheel disc. The effect of pipe friction is taken into account in line with the real world brake dynamics. The resulting brake system model assumes non-laminar flow through restriction as described in the following equation by Fletcher et al. [14]

$$
Q=C_{d} A \sqrt{\frac{2\left(P_{1}-P_{2}\right)}{\rho}} .
$$




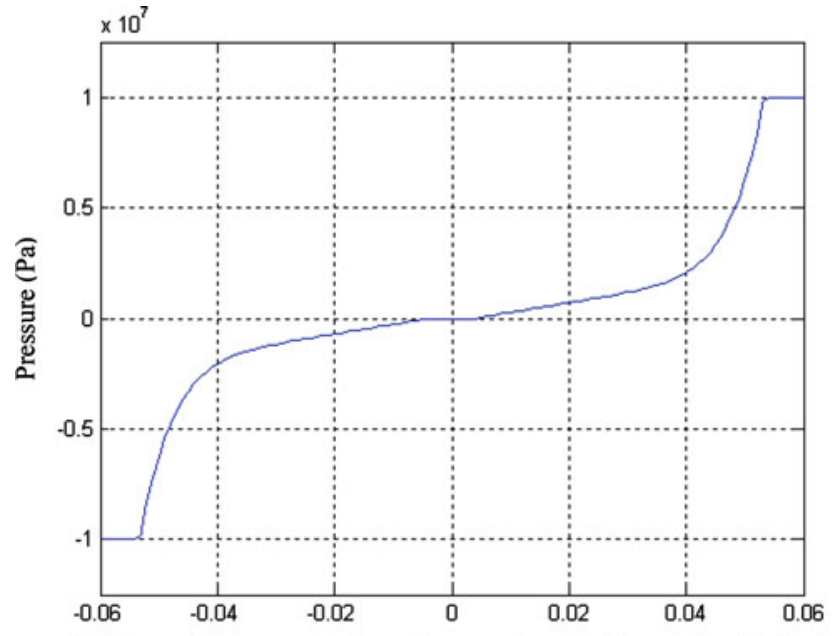

Difference between steering column angle and pinion angle (Radians)

Fig. 4 Hydraulic steering power assist curve

Figure 5 shows a schematic [6] of the hydraulic braking system used in the simulation.

\section{Development of control systems}

\subsection{Electronic stability control (ESC)}

ESC is used to stabilize a vehicle by generating an external yaw moment. The three strategies to perform this are differ- ential braking, active steering and differential drive torque distribution. In this section of the paper, the differential braking, a brake (ABS) based ESC strategy is used. First a fuzzy logic ABS controller is developed and simulated for its performance. Then the ABS controller is extended to develop an ESC controller by additional sensor inputs, like steering angle, yaw rate and sideslip angle and supplemented with an ESC controller algorithm that is capable of enhancing the vehicle stability.

The control architecture as shown in Fig. 6, is designed to be a hierarchical, two layer control [15]. The upper controller has the desired objective of ensuring yaw stability control and assumes that it can command any desired value of yaw torque. The lower controller ensures that the desired value of yaw torque commanded by the upper controller is indeed obtained from the differential braking system based on ABS. The lower controller utilizes the wheel rotational dynamics and controls the braking pressure at each of the four wheels to provide the desired yaw torque for the vehicle. Figure 7 shows one of the control surfaces used to develop the fuzzy logic ESC controller.

The ESC controller used in this paper is developed based on the model reference control technique where the desired vehicle states are generated from a linear 2 DoF reference model (a standard 2 DoF bicycle model with linear tyres). As a function of the vehicle parameters, vehicle longitudinal speed and the steering input, the reference model gen-

Fig. 5 Schematic of the hydraulic brake system
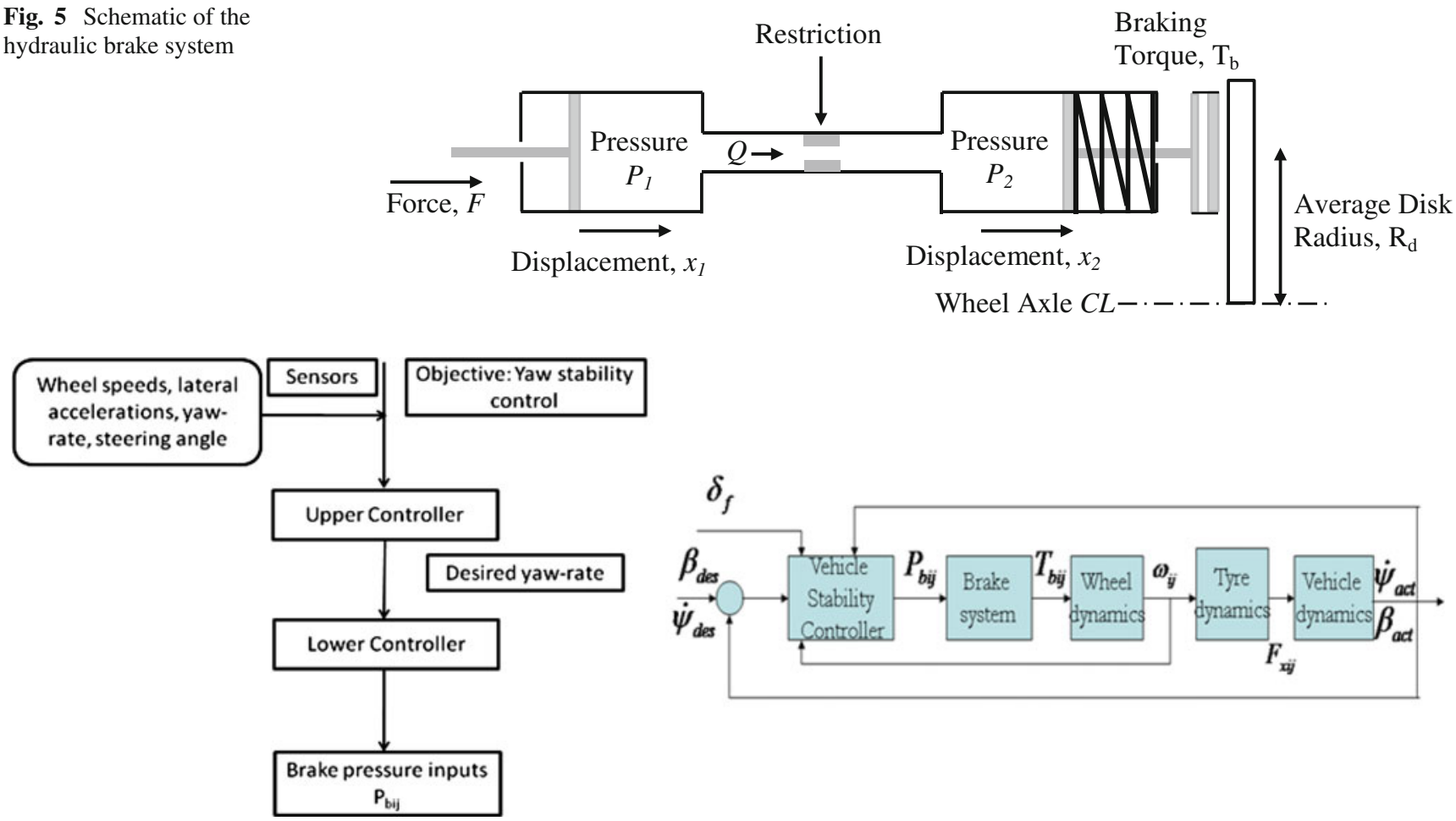

Fig. 6 Schematic of ESC 


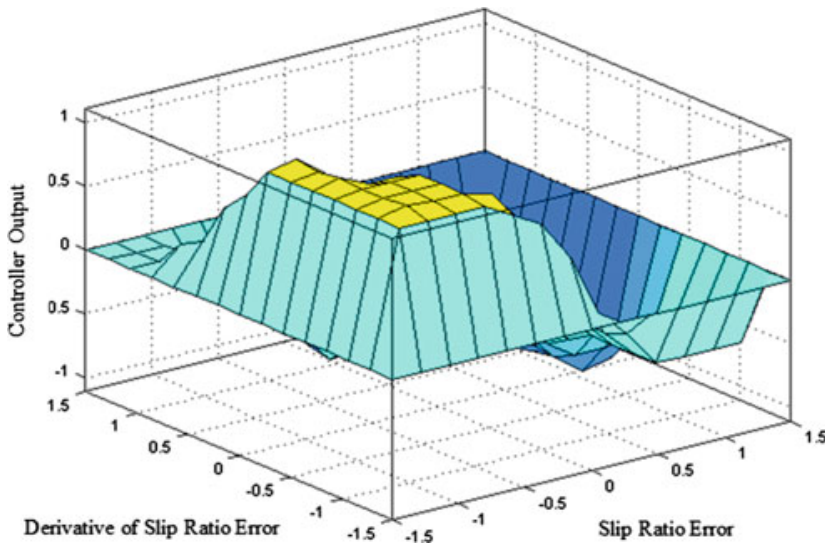

Fig. 7 Control surface of ESC fuzzy controller

erates the desired vehicle state trajectories to be tracked by the actual vehicle. The desired yaw rate can be expressed as shown in the following equation:

$\dot{\psi}_{\text {ref }}=\frac{V_{x}}{L\left(1+\frac{V_{x}^{2}}{V_{c h}^{2}}\right)} \delta_{f}$,

where $\dot{\psi}_{r e f}$ is the reference yaw rate, $V_{c h}$ is the characteristic speed, $L$ is the wheel base, $\delta_{f}$ is the front wheel steering angle, and $V_{x}$ is the longitudinal speed. The characteristic speed $V_{c h}$ in the previous equation can be calculated as follows:

$V_{c h}=\sqrt{\frac{g L}{K_{u s}}}$,

where $K_{u s}$ is the under steering gradient which is a function of the vehicle parameters

$K_{u s}=\frac{m g}{L}\left(\frac{l_{f}}{c_{r}}-\frac{l_{r}}{c_{f}}\right)$,

where $c_{f}$ and $c_{r}$ are the tyre cornering stiffness of the linear tyre model. The calculated desired yaw rate from the above equation is valid only on dry roads with high surface coefficient of friction. The maximum desired yaw rate developed is limited by the surface coefficient of friction. As the surface coefficient decreases the desired yaw rate also decreases. The lateral acceleration is a function yaw rate and the vehicle longitudinal velocity

$a_{y}=\dot{\psi} \times V_{x}$.

The maximum lateral acceleration developed by a vehicle cannot exceed the surface coefficient of friction $\left|a_{y}\right| \leq \mu g$. Taking this into consideration extends the validity of the desired yaw rate calculation. So the maximum desired yaw rate is limited by the following condition: $\dot{\psi}_{\text {limit }} \leq \pm \mu \frac{g}{V_{x}}$.

The same logic is implemented in the desired yaw rate calculation block as follows:

$\dot{\psi}_{\text {des }}=\left\{\begin{array}{ll}\dot{\psi}_{\text {des }}, & \text { if } \quad\left|\dot{\psi}_{\text {des }}\right|<\frac{\mu g}{V_{x}} \\ \frac{\mu g}{V_{x}} \operatorname{sign}\left(\dot{\psi}_{\text {des }}\right), & \text { if } \quad\left|\dot{\psi}_{\text {des }}\right| \geq \frac{\mu g}{V_{x}}\end{array}\right.$.

The calculation of desired side-slip $\beta_{\text {des }}$ angle is made simpler by assuming it to be zero, i.e. $\beta_{d e s}=0$, as driving the vehicle side-slip angle to the minimum increases the vehicle stability. Next, the calculation of the actual vehicle states is carried out. In order to do that, the same steering input $\delta_{f}$ used to calculate the desired state values is also given to the nonlinear vehicle model which generates the actual vehicle states. Then the desired and the actual values of yaw rate and side-slip angle are compared and the errors are used to generate the desired corrective yaw moment.

The fuzzy ESC controller has two inputs, the yaw rate error and side slip angle error and one output the normalised desired corrective yaw moment. This fuzzy controller has an output scaling block which converts ESC controller output to the desired corrective yaw moment. Then the longitudinal brake force required to develop the desired corrective yaw moment is calculated from the kinematics of the brake-tyre force transmission system.

$F_{x i j}=\frac{2 M_{z c}}{t_{i}}$,

where, $t_{i}$ is the track width of the vehicle, $M_{z c}$ is the desired corrective yaw moment. Then the brake pressure required to generate this brake force is calculated as a function of the brake system parameter.

$P_{b i j}=\frac{F_{x i j} r_{w}}{K_{b}}$,

where $r_{w}$ is the radius of the wheel, $K_{b}$ is the brake gain of the brake system and $P_{b i j}$ is the brake pressure. Finally, the allocation of this desired brake pressure on a particular wheel is determined at the lower layer of the ESC controller. This control pressure allocation strategy is based on the direction of steering input (left or right) and the sign of the yaw rate error (under-steer or over-steer).

\subsection{Active front steering (AFS)}

The AFS improves the vehicle dynamics in the lateral direction by extending the linear handling region experienced by the driver in a passive vehicle. In a typical vehicle active steering system, the steering angle at the tyre is set in part by the driver through the vehicle classical steering mechanism while an additional steering angle can be set by the AFS controller using hydraulic or DC motor actuators combined with a differential mechanical device. 
Fig. 8 Schematic of AFS

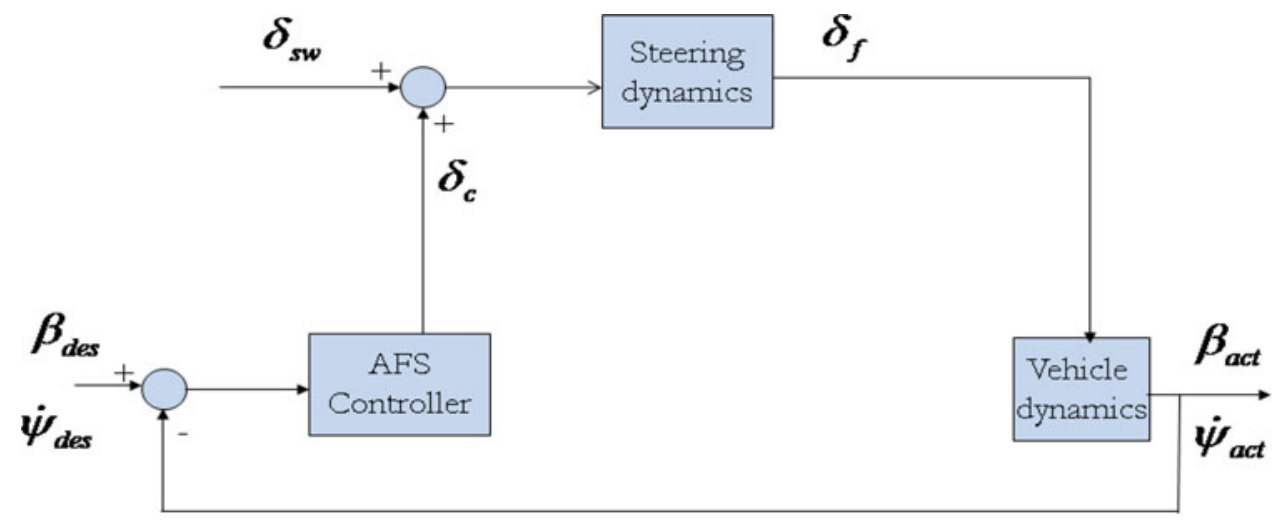

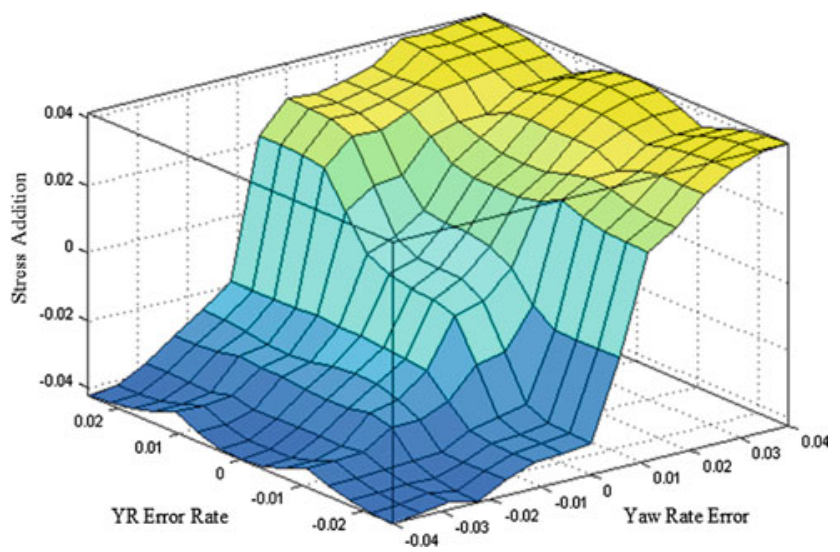

Fig. 9 Control surface of AFS fuzzy controller

The AFS controller used in this paper is a yaw rate error and side slip angle error based fuzzy logic steering controller. The schematic of the AFS control strategy followed in this study described in Fig. 8. The aim of the AFS controller is to minimise the yaw rate and side slip error by modulating the front wheel steer angle, using model reference control technique. The AFS controller receives two inputs the yaw rate and side slip angle errors and provides one output the normalised corrective steering angle. Then an output scaling operation is carried out to convert the normalised steering angle to the required corrective steering angle.

Two commonly used control strategies, PID and fuzzy logic, are used in the development of standalone steering controller in this paper. The vehicle yaw rate and side-slip angle errors (which are the functions of their nominal and actual values respectively) and their time derivatives are fed to the AFS controller to determine the controlled steer addition. Figure 9 shows one of the control surfaces used to develop the fuzzy logic steering controller. Results of earlier research literature in this field are validated here and confirm that performance of AFS is limited within the linear vehicle handling region, i.e., low to medium lateral acceleration range.
3.3 Variable drive torque (VTD)

Another important way to stabilize a vehicle is active drive torque control. One of the recent and widely applied active driveline control techniques is variable drive torque distribution [16]. The objective of this control strategy is to increase vehicle stability and handling capability by suitably distributing the drive torque between wheels. Different drive torque on left and right wheels yield a yaw moment about the vehicle's vertical axis and can be used to stabilize the yaw motion.

The control architecture of the VTD system is hierarchical as used in the ESC controller development in this paper. The upper controller has the objective of ensuring yaw stability control and assumes that it can command any desired value of yaw moment within the capability of the driveline system. The measurement from the wheel speed sensors, yaw rate sensor, an estimation of the vehicle side-slip angle and a steering angle sensor are used. A fuzzy logic control strategy uses these measurements and computes the desired value of the corrective yaw moment. The lower controller ensures that the desired value of yaw torque commanded by the upper controller is indeed obtained from the torque management system. The lower controller uses the driveline dynamics and controls the biasing of the drive torque management system to provide the desired yaw torque for the vehicle. A PI controller strategy is followed in this case and developed in Matlab/Simulink. The PI controller takes the yaw rate and side-slip angle errors as inputs and returns a control value between 0 and 1 giving the ratio of the drive torque transmitted to the left and right wheels. The control architecture of VTD used in this paper is shown in Fig. 10.

\subsection{Active suspension normal force control (NFC)}

Active suspension in this paper is another active vehicle control system that minimizes the longitudinal and lateral load transfer between the wheels. The NFC model used in this research has hydraulic actuators at each wheel as shown in Fig. 11 that either add or subtract an extra force on each 
Fig. 10 Schematic of VTD control

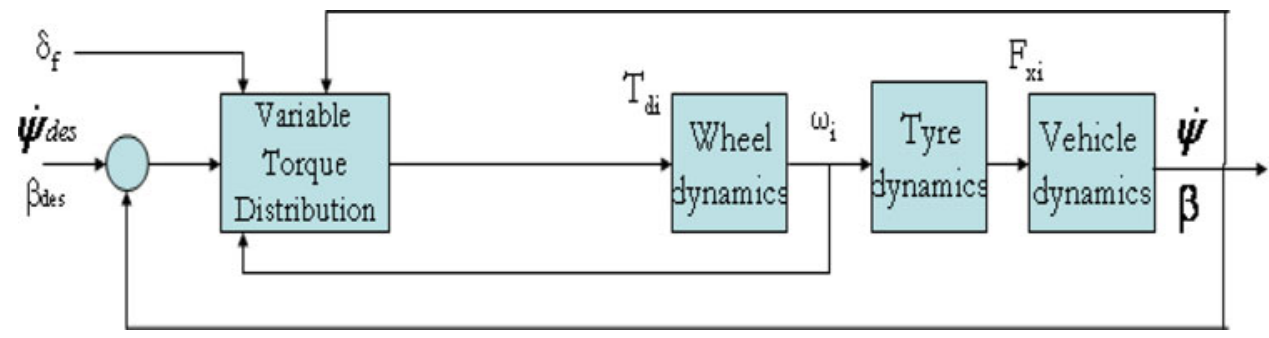

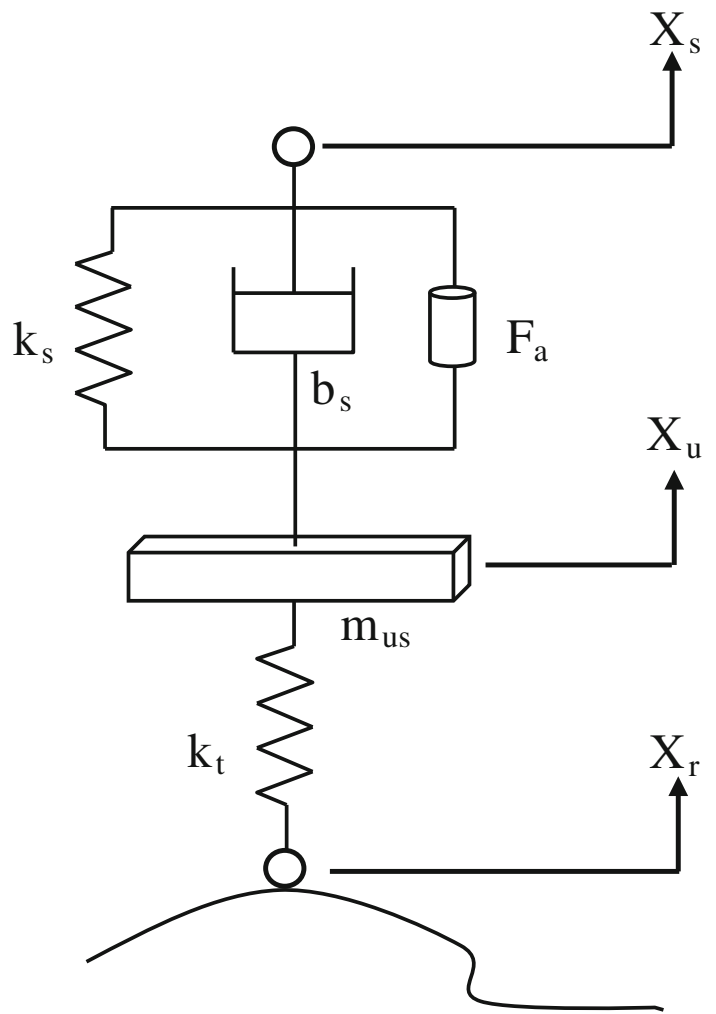

Fig. 11 Quarter car suspension model

wheels and designed to optimize the normal forces on wheels based on signals from the active suspension controller as a function of various vehicle dynamic states. It ensures the tracking of the desired suspension force using PID and fuzzy logic control strategies. Considerable literature can be found on the dynamics and control of hydraulic actuators for active automotive suspensions.
The hydraulic actuator dynamics used in this paper includes the dynamics of a spool valve controlled hydraulic actuator model explained by Rajamani and Hedrick [13]. The schematic of the active suspension control strategy followed is described in Fig. 12 and few of the control surfaces used to develop the fuzzy logic suspension controller are shown in Fig. 13.

For the purpose of this paper, the suspension control strategy used has the following objectives:

- To add the required amount of active suspension forces at the individual wheel corners to reduce the vehicle yaw rate and side-slip angle.

- To reduce or maintain roll angle compared to a passive vehicle.

The NFC controller strategy used in this paper is a yaw rate and side slip angle errors based fuzzy logic normal force controller. The main aim of the NFC controller is to minimise the yaw rate and side slip angle error by modulating the front tyre normal forces, using fuzzy feedback control strategy. The NFC controller receives two inputs the yaw rate and side slip angle errors and provides two outputs the normalised active suspension control force. Then an output scaling operation is carried out to convert the normalised active suspension control forces to the required corrective suspension normal forces.

\section{Analysis of standalone systems}

The active chassis control systems can be classified as standalone systems if each system has its own sensor(s), controller and actuator(s) modules. The standalone systems do
Fig. 12 Schematic of active suspension (NFC) system

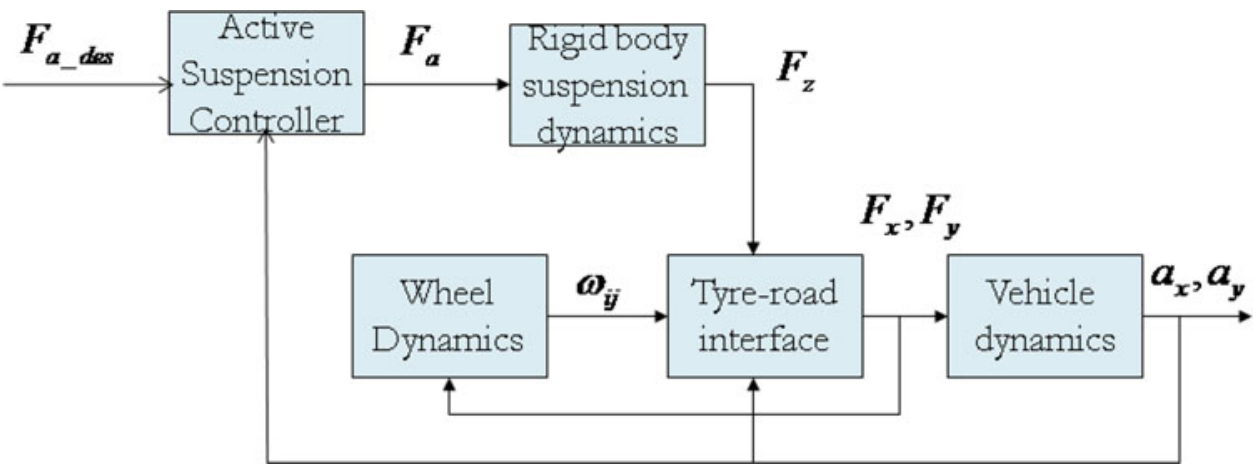



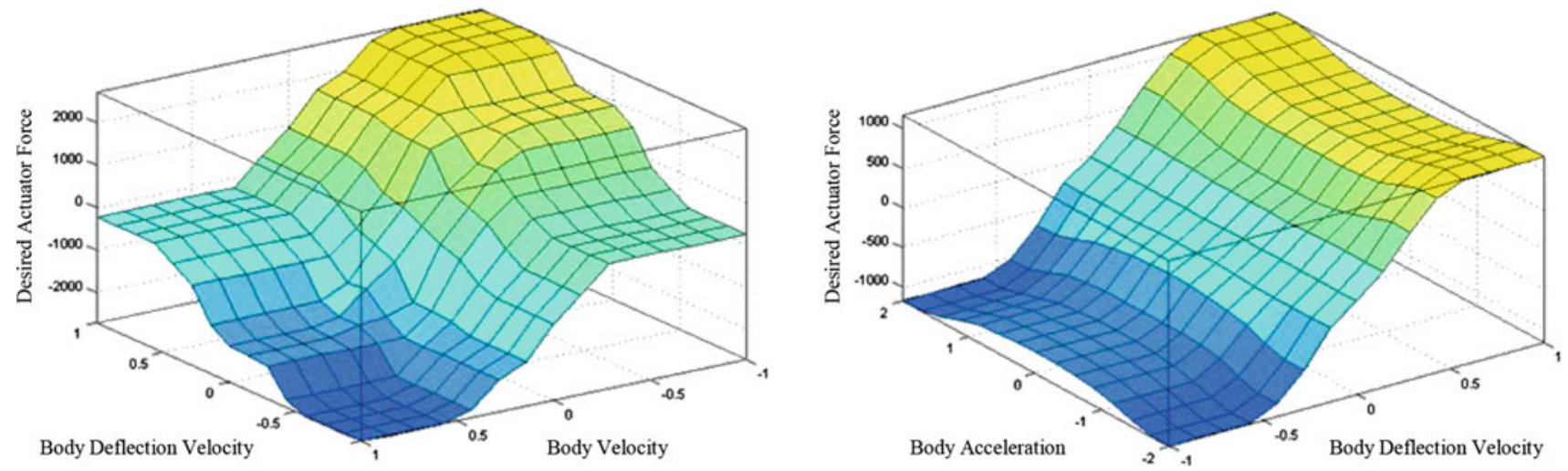

Fig. 13 Control surface of NFC fuzzy controller

not interact with each other in terms of resources and information sharing. And they also individually try to achieve their own control objective(s) without taking into account whether it affects the control objective(s) of other active systems or not.

The section to analyse the standalone control systems is divided into four subsections one for each active chassis system in consideration. The overall aim of all these systems is to improve the vehicle stability by reducing the yaw rate and side slip angle, but they achieve it through different methods, such as controlling the distribution of braking, driving, steering and suspension forces. Studying and analysing the ability of each of these four systems in developing their control outputs will highlight their individual control authority in improving vehicle handling.

\subsection{Electronic stability control}

Results of the earlier research show that the brake based ESC are more effective in a wide lateral acceleration (latac) range is validated through simulations. The control authority of electronic stability control system are analysed by running the vehicle model on dry, wet and icy road conditions at $0.2 \mathrm{~g}$ and $0.3 \mathrm{~g}$ for the low latac, $0.4 \mathrm{~g}, 0.5 \mathrm{~g}$ and $0.6 \mathrm{~g}$ for the medium latac and at $0.7 \mathrm{~g}$ and $0.8 \mathrm{~g}$ for the high latac operating ranges, respectively. The control authority of ESC at the handling limits is also investigated. From the simulation results, as shown in Figs. 14 and 15, it can be seen that ESC improves the vehicle handling by reducing the peak yaw rate by $12 \%$ at $0.2 \mathrm{~g}$ and by $9 \%$ at $0.3 \mathrm{~g}$ latac on a dry road. Similarly, a $23 \%$ reduction in the peak slip angle is obtained at $0.2 \mathrm{~g}$ and a
Fig. 14 Intrusive nature of ESC on longitudinal dynamics in low latac

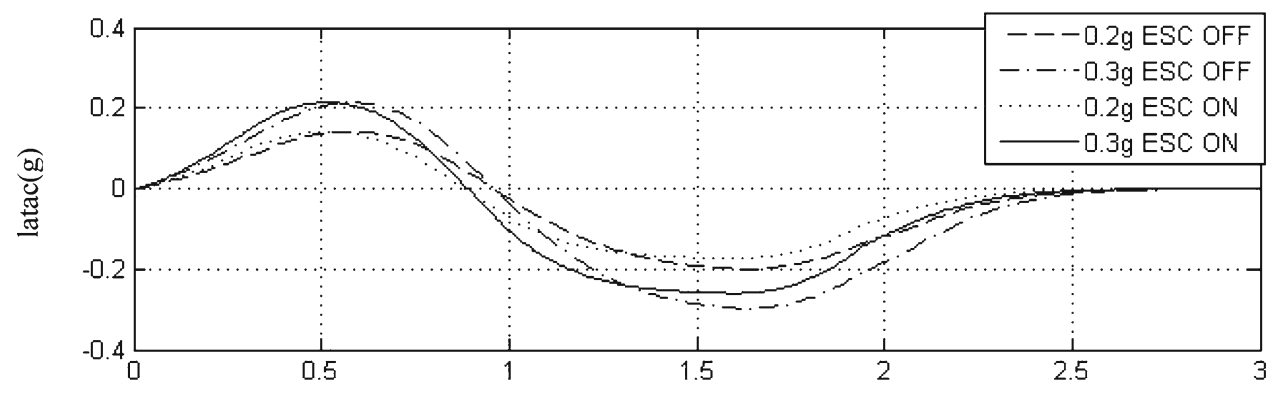

Time(s)

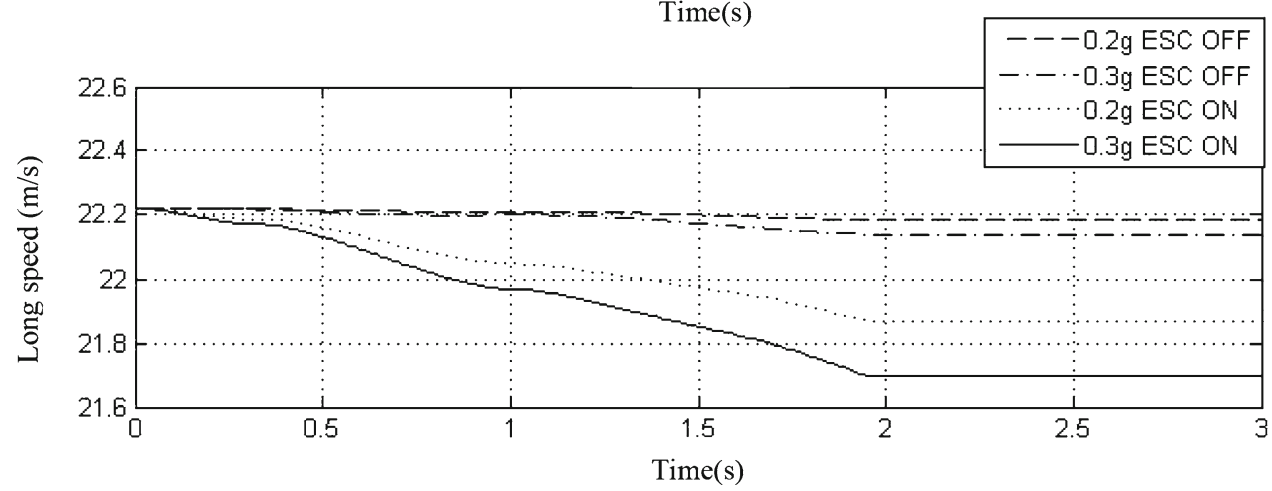


Fig. 15 Control authority of ESC during low latac
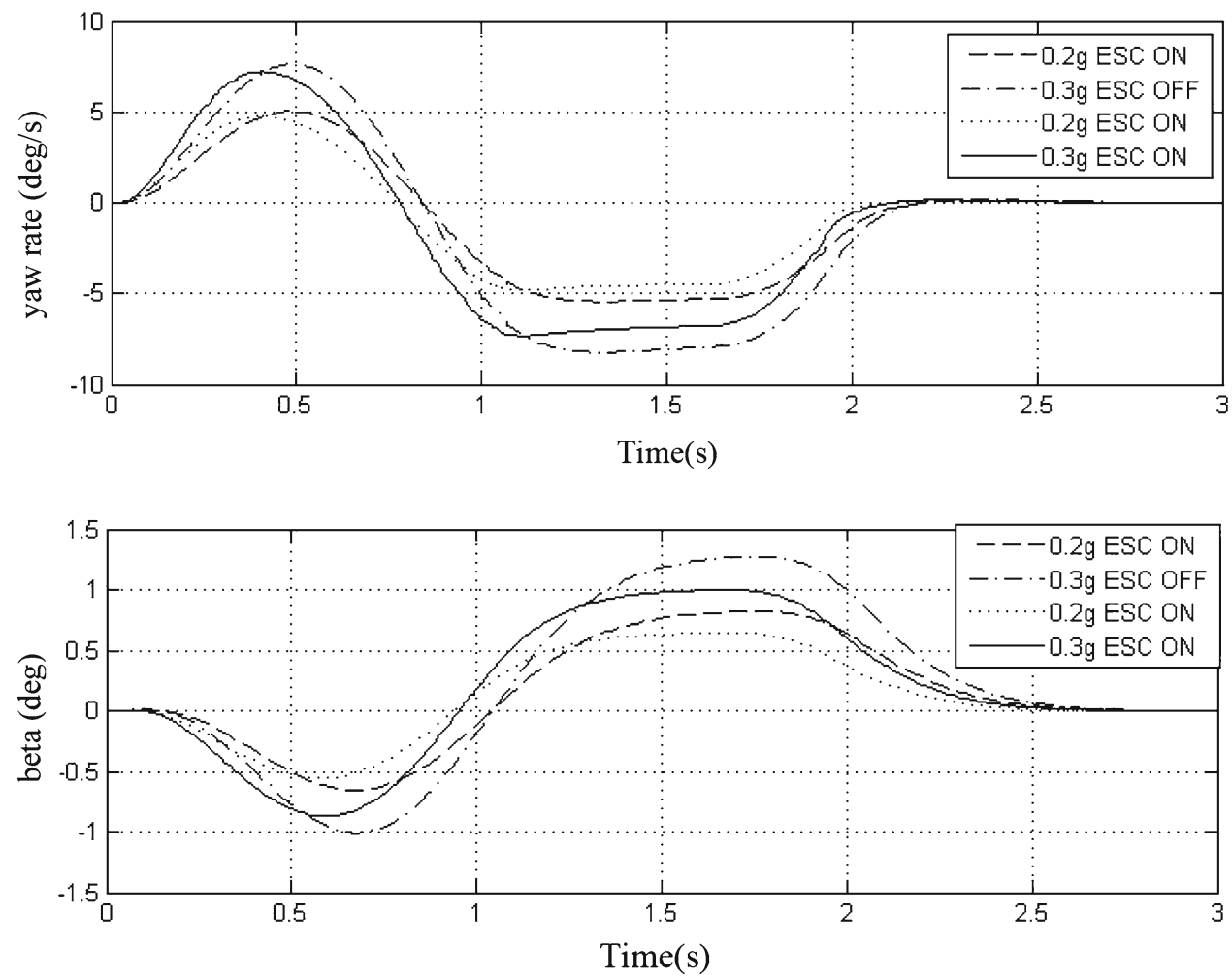

$20 \%$ reduction at $0.3 \mathrm{~g}$. One important observation due to the activation of ESC controller is that it reduced the longitudinal vehicle speed by $1.4 \%$ at $0.2 \mathrm{~g}$ and by $2.0 \%$ at $0.3 \mathrm{~g}$ latac. This highlights the intrusive nature of this control system in the longitudinal dynamics of the vehicle. This is generally not a preferable characteristic from a vehicle in driver's point of view, especially in the low lateral acceleration range which is not a safety critical operating range.

In the medium latac range, the ESC improves the vehicle handling by reducing the peak yaw rate by $12 \%$ at $0.4 \mathrm{~g}$, by $10 \%$ at $0.5 \mathrm{~g}$ and by $6 \%$ at $0.6 \mathrm{~g}$ latac on a dry road. Similarly a $22 \%$ reduction in the peak slip angle is obtained at $0.4 \mathrm{~g}$, $21 \%$ at $0.5 \mathrm{~g}$ and a $20 \%$ reduction at $0.6 \mathrm{~g}$. And it is observed that the activation of ESC controller reduces the longitudinal vehicle speed by $2.5 \%$ at $0.4 \mathrm{~g}, 2.95 \%$ at $0.5 \mathrm{~g}$ and by $3.2 \%$ at $0.6 \mathrm{~g}$ latac. This is again not a preferable characteristic from a vehicle in driver's point of view in the medium lateral acceleration range which is not a safety critical operating range.

In the high latac range ESC improves the vehicle handling by reducing the peak yaw rate by $12 \%$ at $0.7 \mathrm{~g}$ and by $9.6 \%$ at $0.8 \mathrm{~g}$ latac on a dry road. Similarly a $23 \%$ reduction in the peak slip angle is obtained at $0.7 \mathrm{~g}$ and a $27 \%$ reduction at $0.8 \mathrm{~g}$. One important observation due to the activation of ESC controller is that it reduced the longitudinal vehicle speed by $1.4 \%$ at $0.7 \mathrm{~g}$ and by $2.9 \%$ at $0.8 \mathrm{~g}$ latac. This highlights the intrusive nature of this control system in the longitudinal dynamics of the vehicle. This is generally not a preferable characteristic from a vehicle in driver's point of view, especially in the low lateral acceleration range which is not a safety critical operating range.

\subsection{Active front steering}

The control authority of AFS system are analysed by running the vehicle model on dry, wet and icy roads at $0.2 \mathrm{~g}$ and $0.3 \mathrm{~g}$ for the low lateral acceleration (latac), $0.4 \mathrm{~g}, 0.5 \mathrm{~g}$ and $0.6 \mathrm{~g}$ for the medium latac and at $0.7 \mathrm{~g}$ and $0.8 \mathrm{~g}$ for the high latac operating ranges respectively. The control authority of AFS at the handling limits is also investigated. From the simulation results, as shown in Figs. 16 and 17, it can be seen that AFS improves the vehicle handling by reducing the peak yaw rate by $14 \%$ at $0.2 \mathrm{~g}$ and by $8 \%$ at $0.3 \mathrm{~g}$ latac on a dry road.

Similarly, a $17 \%$ reduction in the peak slip angle is obtained at $0.2 \mathrm{~g}$ and a $15 \%$ reduction at $0.3 \mathrm{~g}$. Similarly, in the medium latac range, the AFS improves the vehicle handling by reducing the peak yaw rate by $17 \%$ at $0.4 \mathrm{~g}$, by $10 \%$ at $0.5 \mathrm{~g}$ and by $7 \%$ at $0.6 \mathrm{~g}$ latac on a dry road. Similarly a $17 \%$ reduction in the peak slip angle is obtained at $0.4 \mathrm{~g}$, $20 \%$ at $0.5 \mathrm{~g}$ and a $20 \%$ reduction at $0.6 \mathrm{~g}$. Again the AFS does not affect the longitudinal vehicle speed at the end of the manoeuvre and the longitudinal vehicle speed is at par with the passive vehicle at $0.4 \mathrm{~g}$ and better by $0.5 \%$ at $0.5 \mathrm{~g}$. This highlights the non-intrusive nature of this control system in the longitudinal dynamics of the vehicle in the medium latac range as well. 
Fig. 16 Control authority of AFS at $0.2 \mathrm{~g}$ on dry road conditions

Fig. 17 Influence of AFS on longitudinal dynamics at $0.2 \mathrm{~g}$
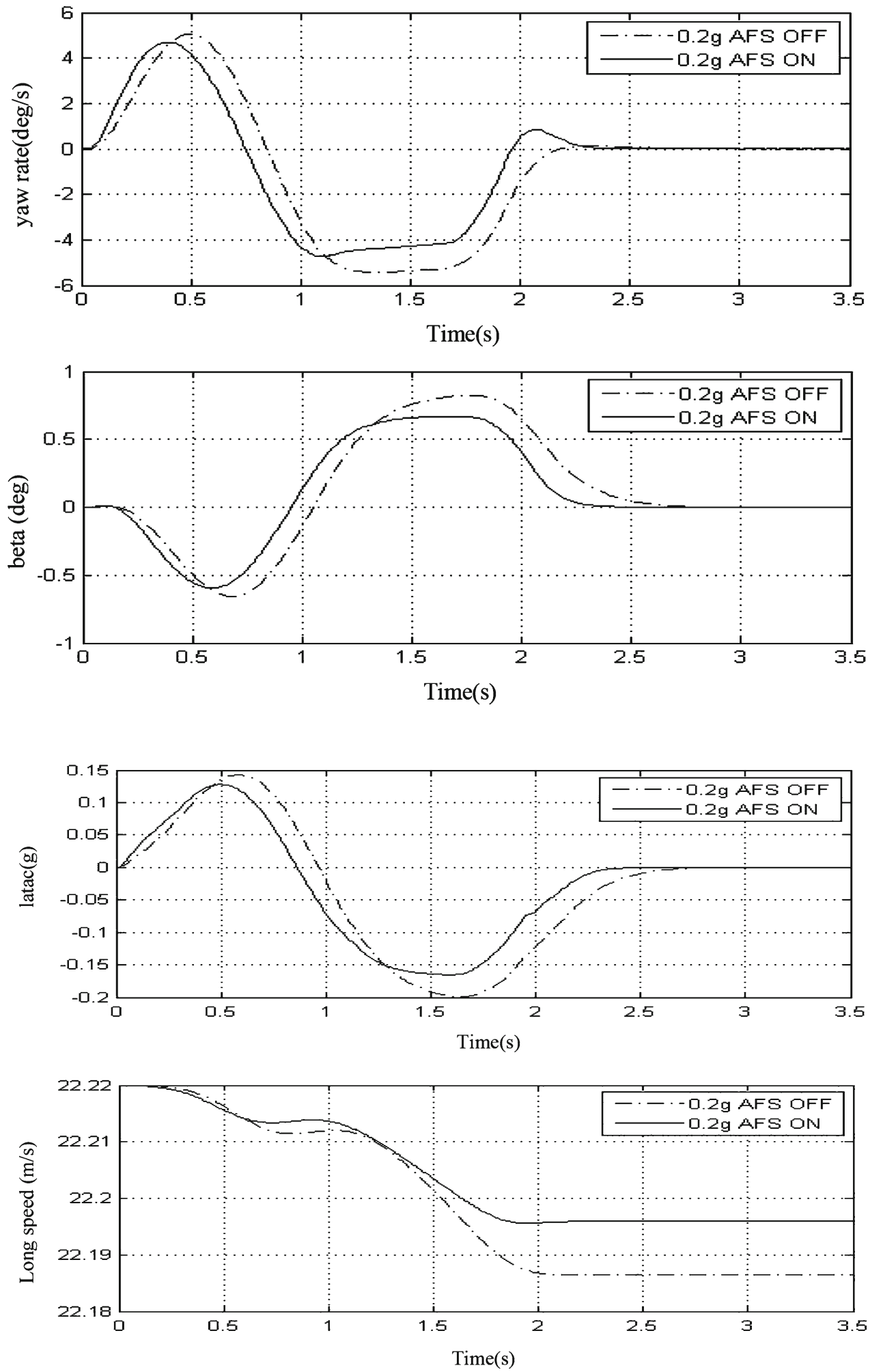

In the high latac range AFS improves the vehicle handling by reducing the peak yaw rate by $4 \%$ at $0.7 \mathrm{~g}$ and by $4 \%$ at $0.8 \mathrm{~g}$ latac on a dry road. Similarly an $18 \%$ reduction in the peak slip angle is obtained at $0.7 \mathrm{~g}$ and a $25 \%$ reduction at $0.8 \mathrm{~g}$. Again the AFS does not affect the longitudinal vehi- cle speed at the end of the manoeuvre and the longitudinal vehicle speed is better by $0.8 \%$ at $0.7 \mathrm{~g}$ and by $1.8 \%$ at $0.8 \mathrm{~g}$. This highlights the non-intrusive nature of this control system in the longitudinal dynamics of the vehicle in the high latac range. 


\subsection{Variable drive torque distribution}

The control authority of VTD system are analysed by running the vehicle model on dry, wet and icy roads at the low, medium and the high latac operating ranges respectively. The control authority of VTD at the handling limits is also investigated. From the simulation results, as shown in Figs. 18, 19 and 20, it can be seen that VTD improves the vehicle handling by reducing the peak yaw rate by $12 \%$ and the peak slip angle by $28 \%$ in the low latac region on a dry road. In the medium latac range, the VTD improves the vehicle stability by reducing the peak yaw rate by $11 \%$ and the peak slip angle by $20 \%$. A $6 \%$ peak yaw rate improvement and $37 \%$ peak side slip angle improvement is obtained with VTD against a passive vehicle.

One important observation due to the activation of VTD controller is that, due to addition of driving torque at the wheels to improve yaw rate tracking and stability the reduction in the exit speed is less compared to the ESC and AFS vehicles. And the reduction in the exit speed at high latac region is much more pronounced than at the low and medium latac region, but still much better than the passive vehicle. Unlike the brake based ESC system, VTD does not intrude with the vehicle's longitudinal dynamics. This is a much more preferable characteristic from a driver's point of view, especially in the low lateral acceleration range which is not a safety critical operating range.

\subsection{Active suspension}

The control authority of suspension NFC system on vehicle handling are analysed by running the vehicle model on dry, wet and icy roads at the low, medium and the high latac operating ranges respectively. From the simulation results, as shown in Figs. 21, 22 and 23, it can be seen that when NFC is activated in the low latac region it does improve the vehicle handling by reducing the peak yaw rate and the peak slip angle but the improvement is negligible. This is because the lateral load transfer between the outer and inner wheels is not very large during low latac. And also the control strategy optimises the addition of suspension normal force as a function of the vehicle roll angle which is reduced by the controller. But we can observe an improvement in this trend with more reduction in the peak yaw rate and the peak side slip angle as the vehicle moves into the medium latac zone.

The superiority of the active system continues in the high latac range as well but with a diminishing effect on the control authority. In all the three latac regions a good roll control is obtained except at the limits. The main reason for this behaviour of the NFC system is that, at low latac, the tyre is operating at its linear region and hence producing lateral force as a function of the slip angle and the normal wheel load. Being operated at the same slip angle, between passive and active vehicles with little effect on lateral load transfer reduction by NFC system, the output lateral tyre force produced
Fig. 18 Control authority of VTD at low latac
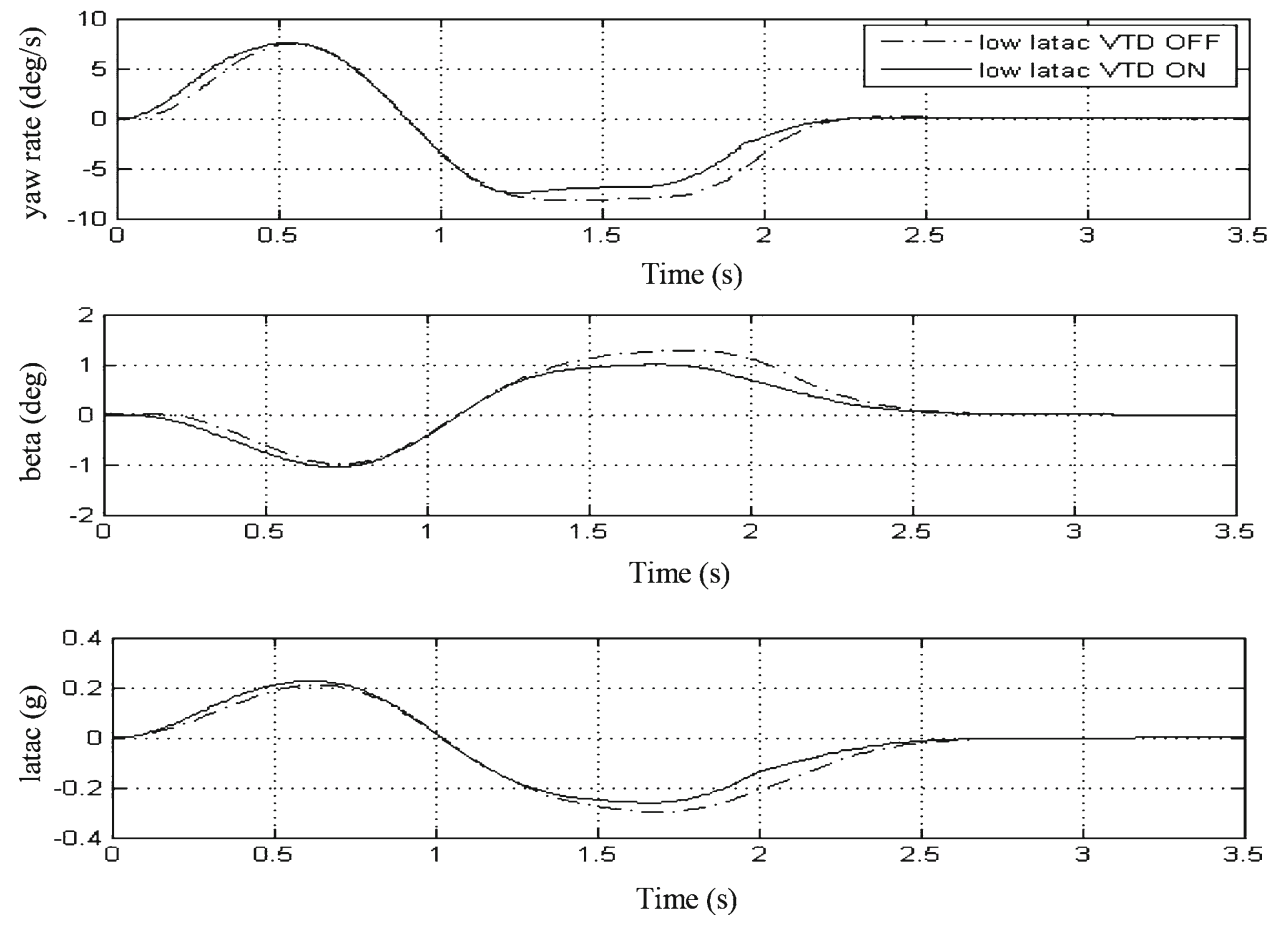
Fig. 19 Control authority of VTD at medium latac
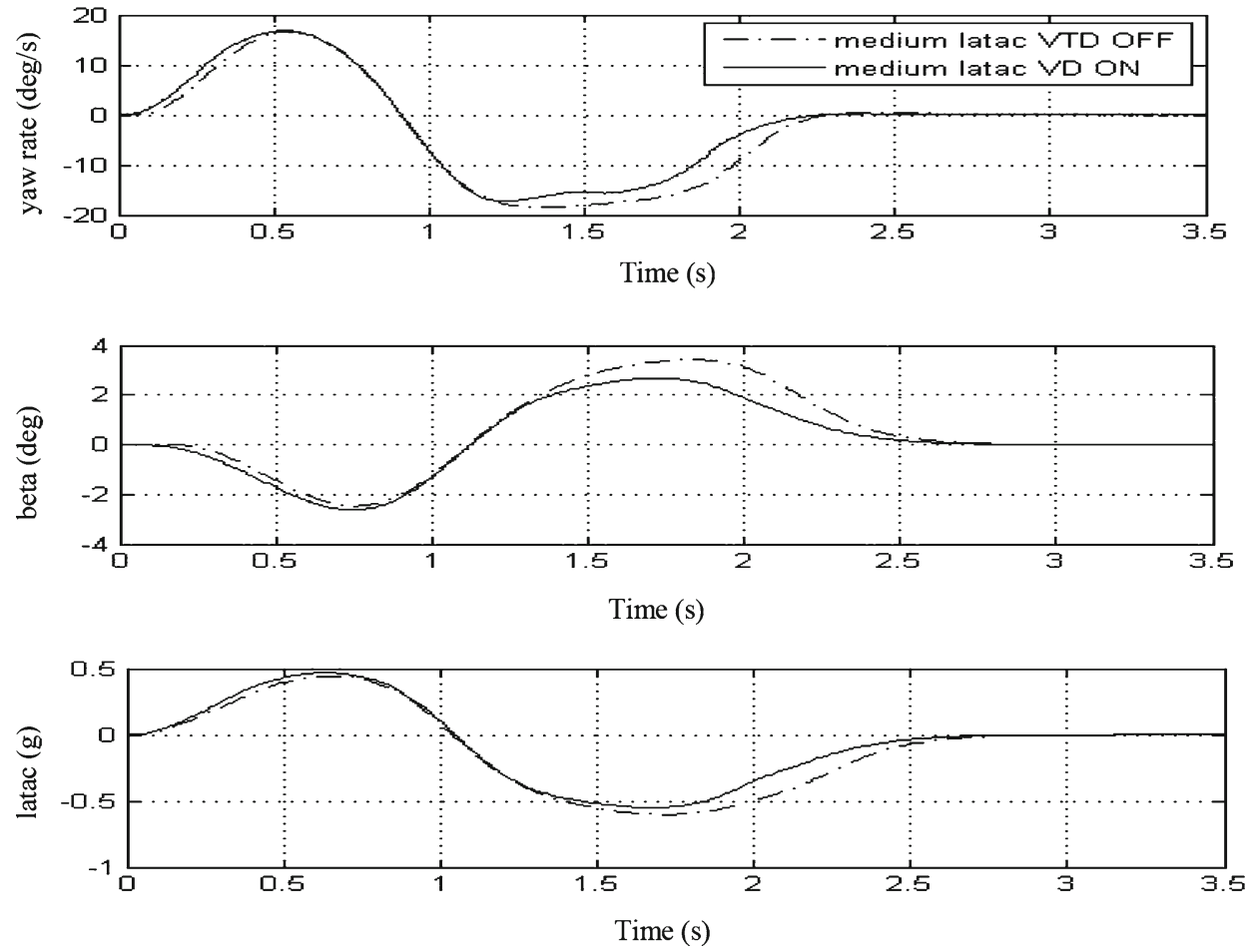

Fig. 20 Control authority of VTD at high latac
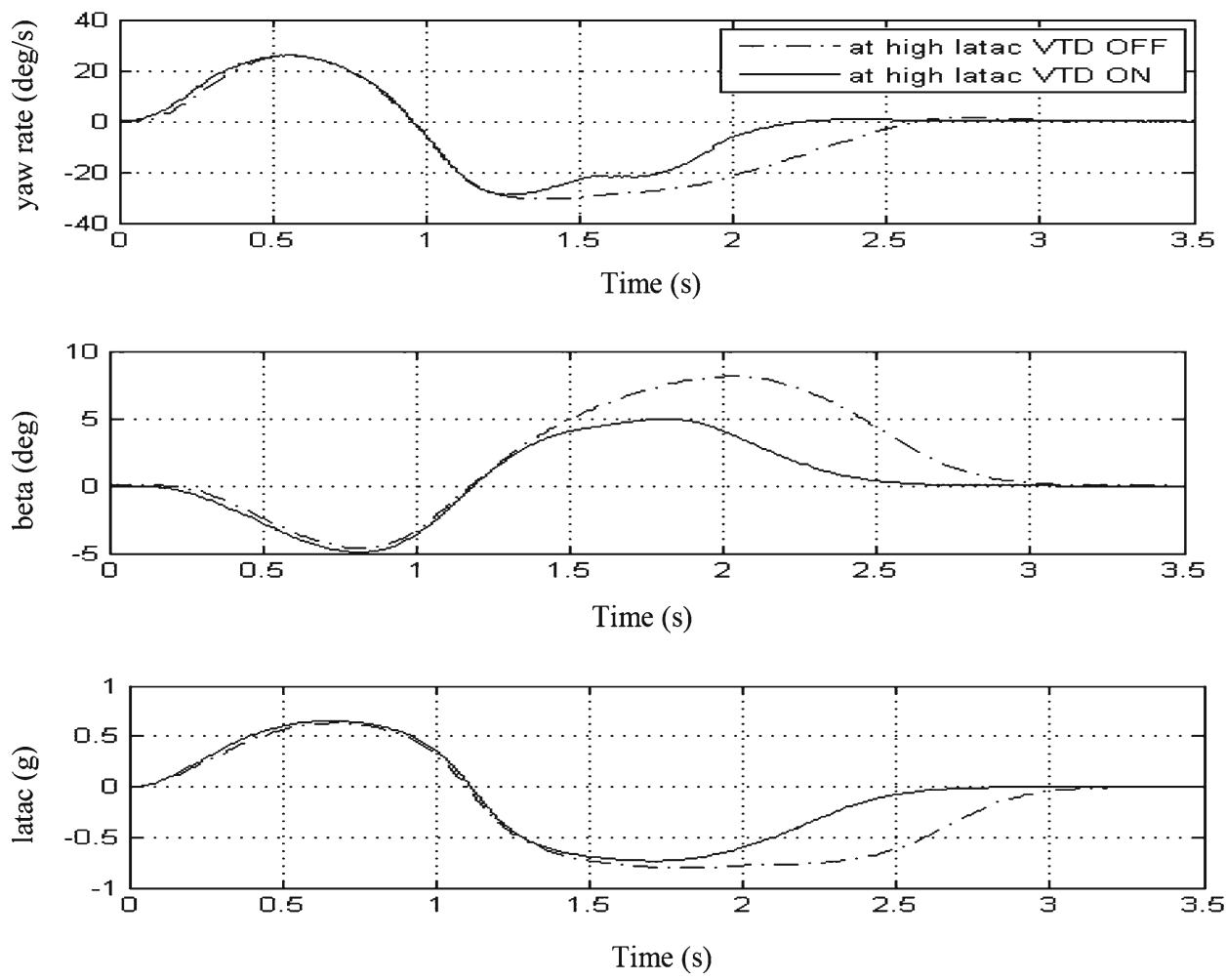

by the active system provides a negligible improvement in the reduction of yaw rate and slip angle. But at the medium latac zone, supported with a more reduction in lateral load transfer the NFC system produces better handling compared to a passive vehicle.
Again, at the high latac, the trend continues but with a reduced efficiency due to the addition of more active suspension normal force results in a tyre normal load instability that affects the effective generation lateral and longitudinal forces. This limits the extent / capacity of the normal suspen- 
Fig. 21 Control authority of NFC at low latac

Fig. 22 Control authority of NFC at medium latac
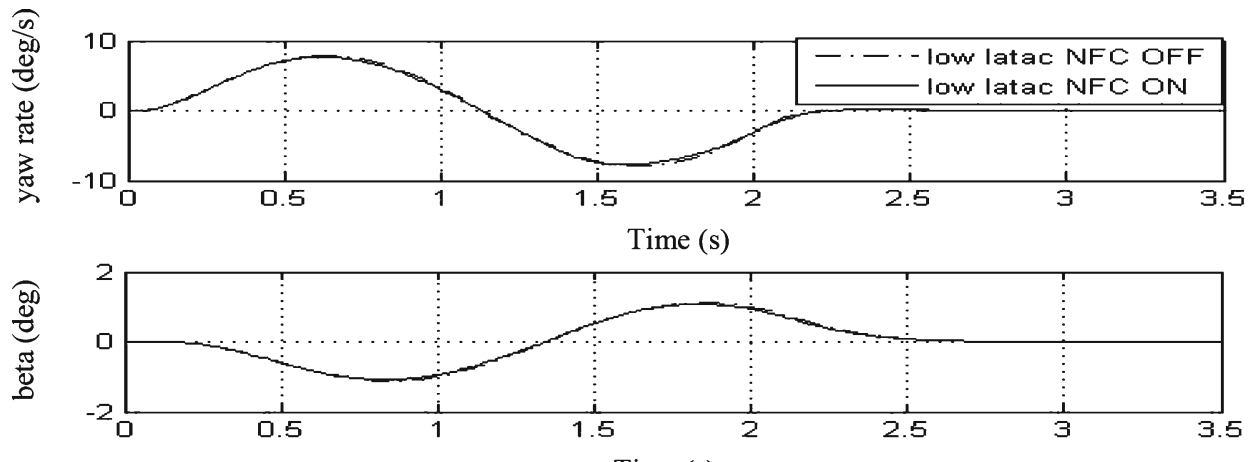

Time (s)
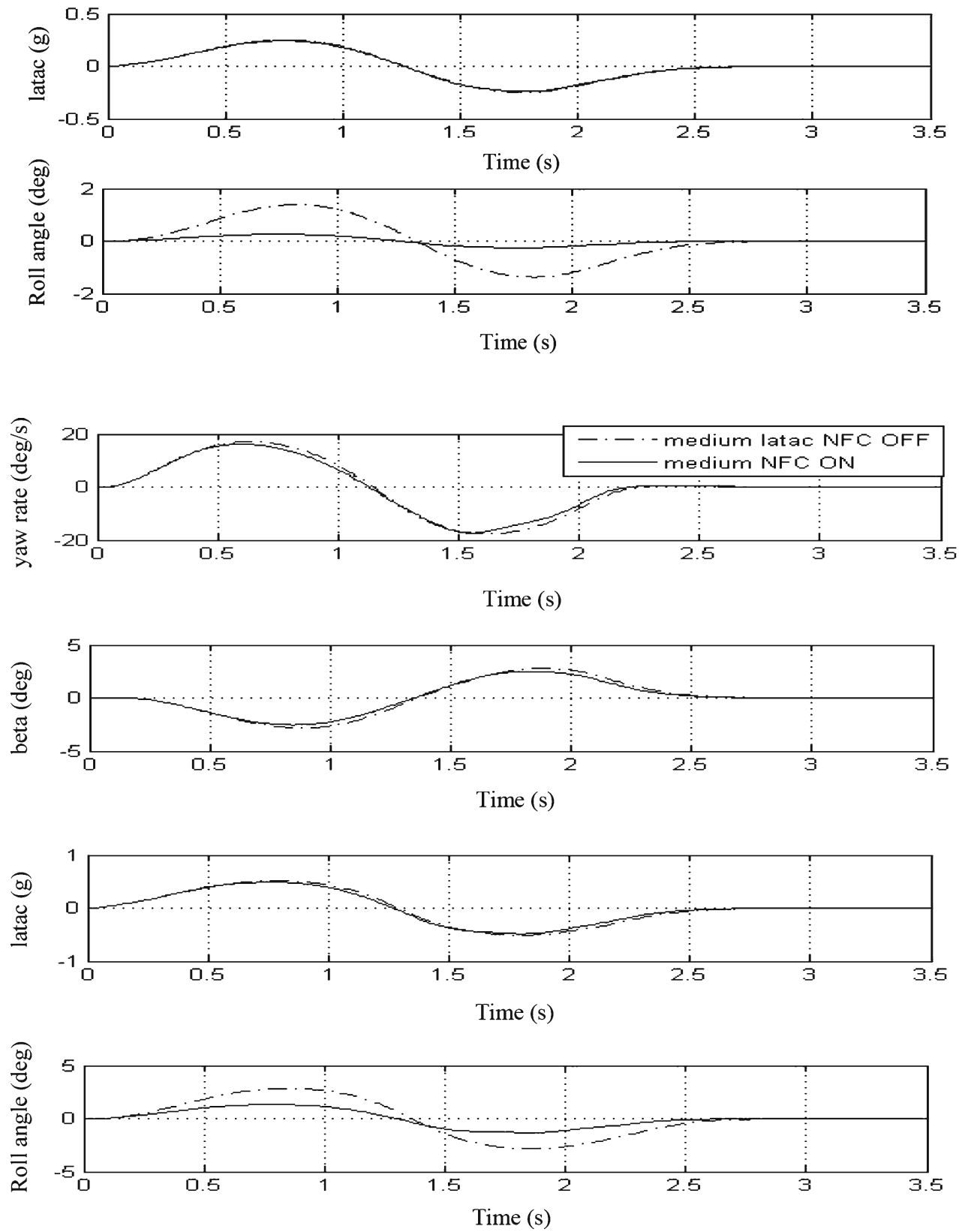
Fig. 23 Control authority of NFC at high latac
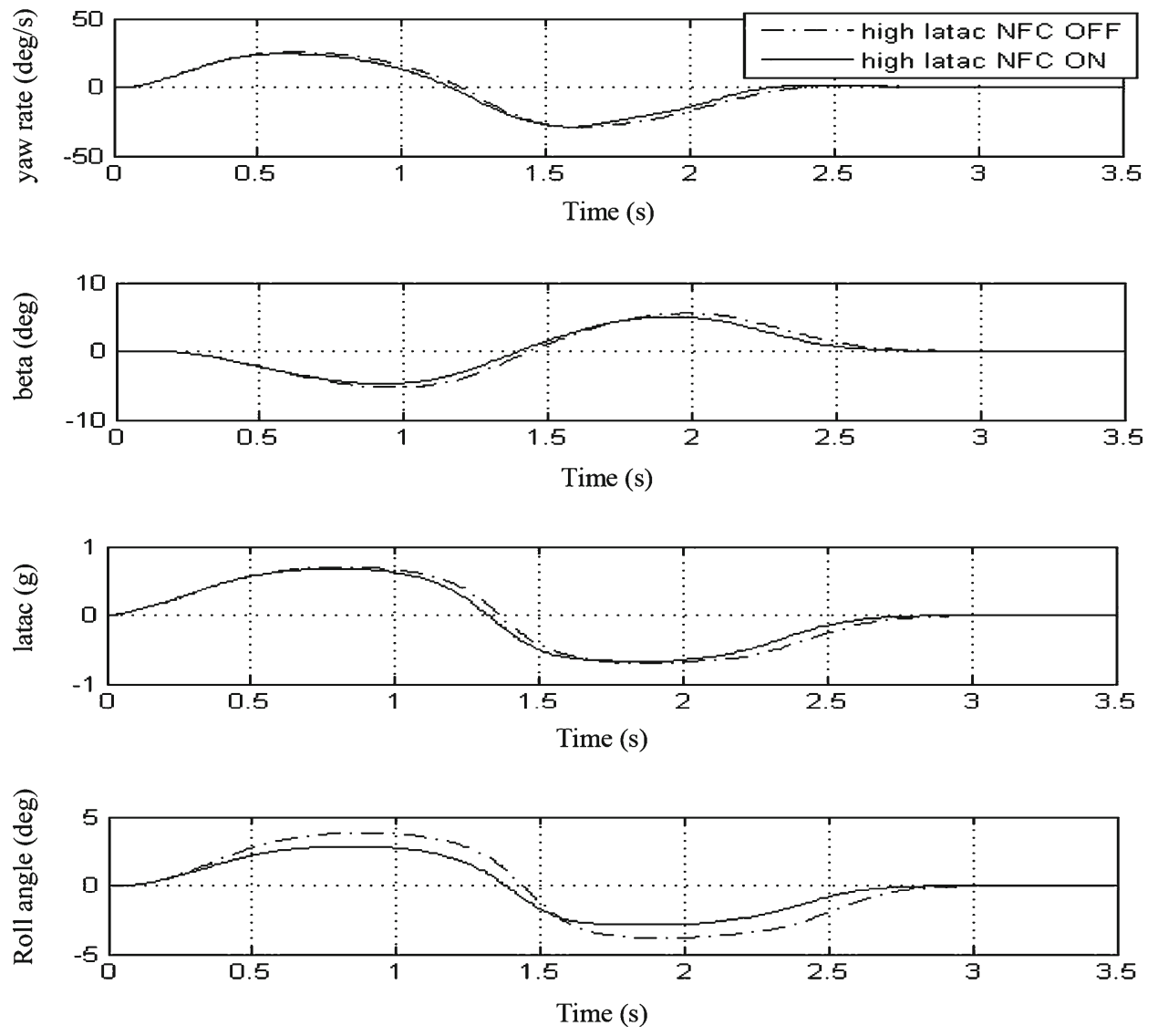

Fig. 24 Schematic of AFS + ESC standalone controller

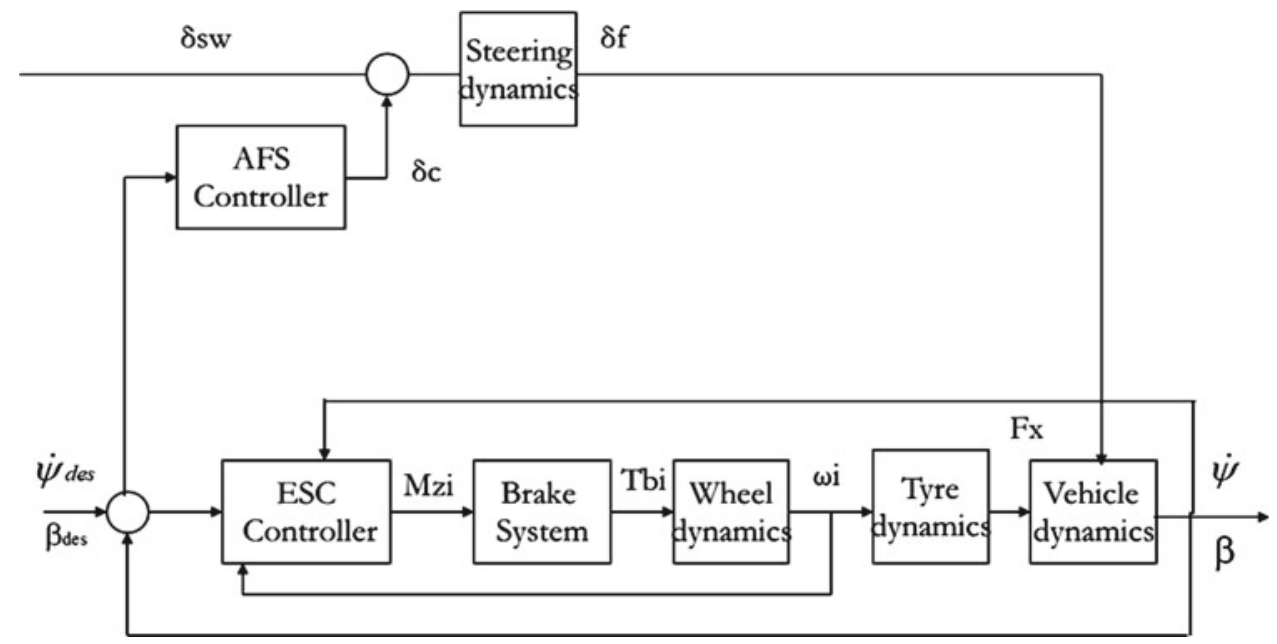

sion force actuator. So it is evident that the NFC does have the capability to improve the vehicle stability at the medium latac but its control authority is limited and diminished at low and high latacs respectively. At the limits, the NFC ceases to display any ability to improve the vehicle handling than the passive vehicle.

\section{Integrated control systems}

5.1 Rule based integrated control strategy

Hence, in order to avoid undesirable interactions between control subsystems and reduce performance trade-offs in 
Fig. 25 Low latac performance of AFS + ESC in standalone mode

Fig. 26 Schematic of AFS + ESC integrated controller (ICC)
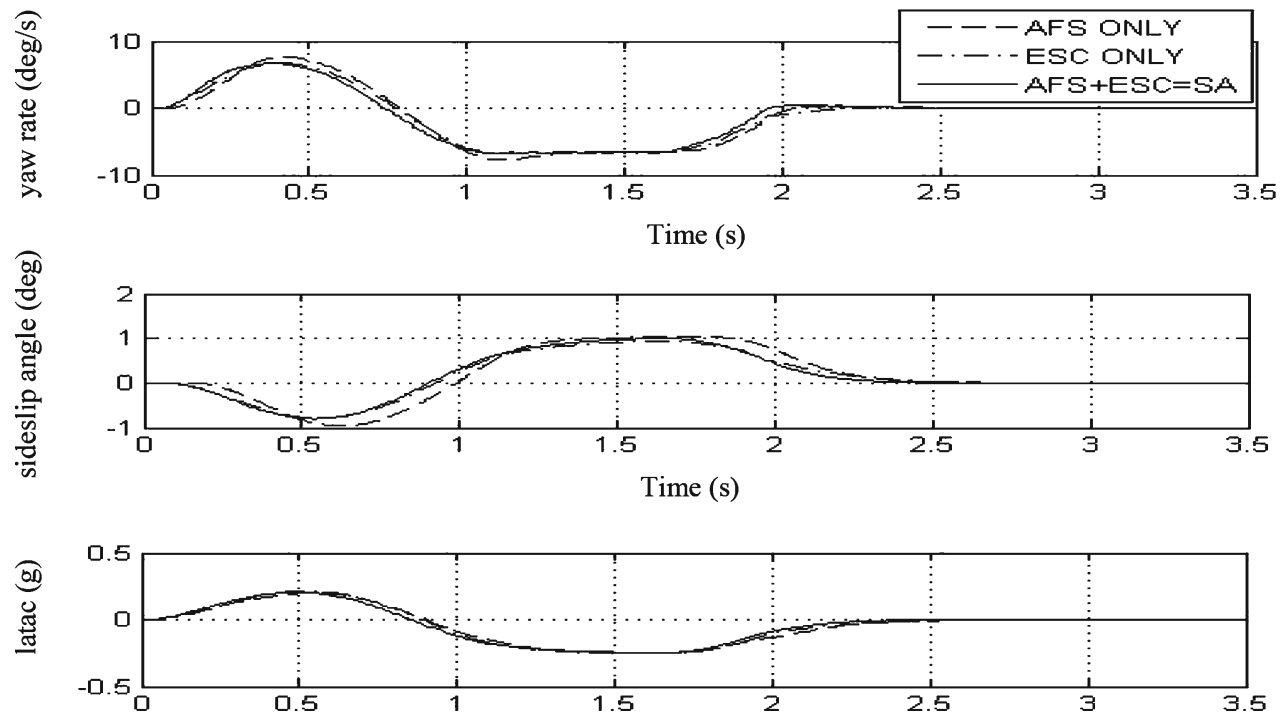

Time (s)

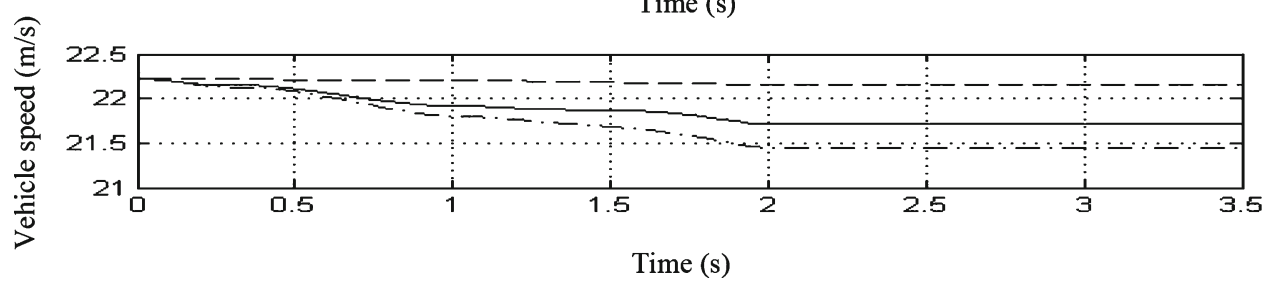

Time (s)

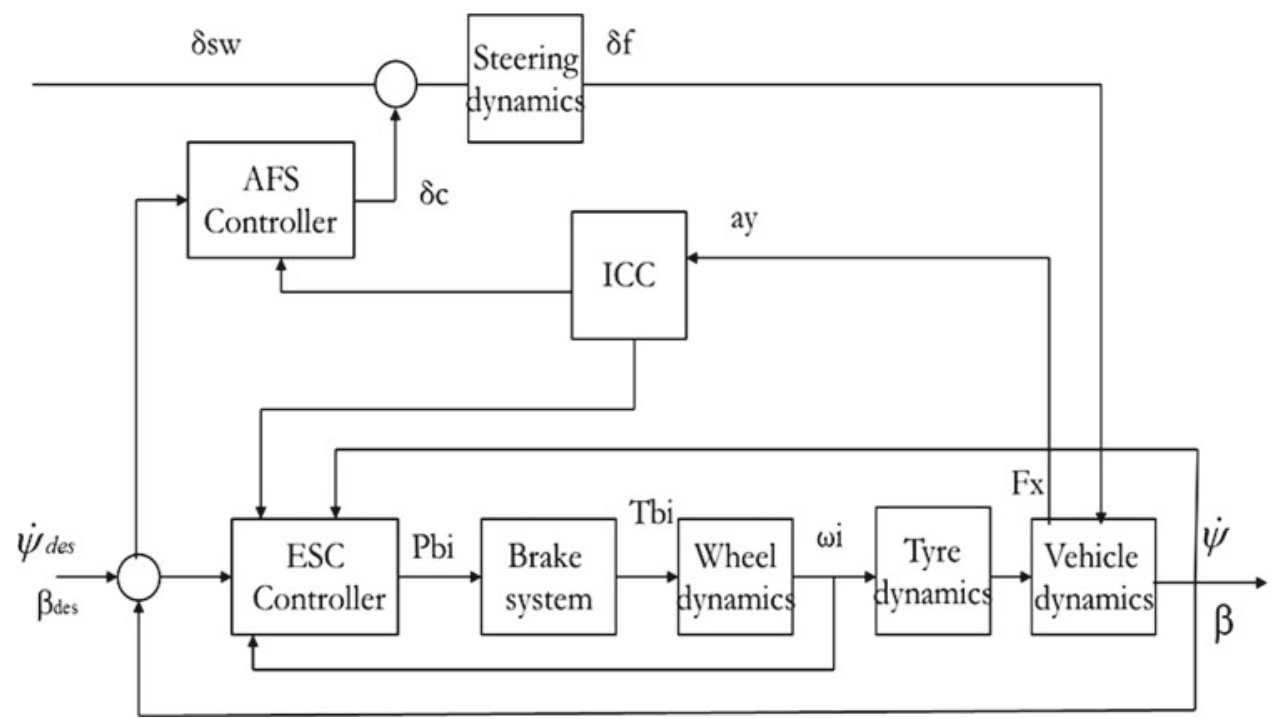

vehicle handling, a new rule based integration scheme is proposed to coordinate the control actions of the stand-alone controllers. In light of the previous analysis of stand-alone active subsystems, the proposed integrated control system will be designed to achieve the following objectives:

- To improve vehicle steerability at low to mid-range lateral accelerations.

- To maintain vehicle stability close to and at the limit of handling.
- To minimize the influence of brake intervention on the longitudinal vehicle dynamics.

This strategy needs to determine the activation sequences and active regions of the stand-alone controllers in terms of the current vehicle operating point to avoid conflicts and to enhance the coexistence. It is therefore necessary to measure the vehicle operating point. The operating point of the vehicle ranges from normal driving to limit handling. A quantitative measure of this is the lateral acceleration of the vehicle. 
Fig. 27 Performance of ICC (AFS + ESC) at medium latac

Fig. 28 Performance of ICC $(\mathrm{AFS}+\mathrm{ESC})$ at high latac
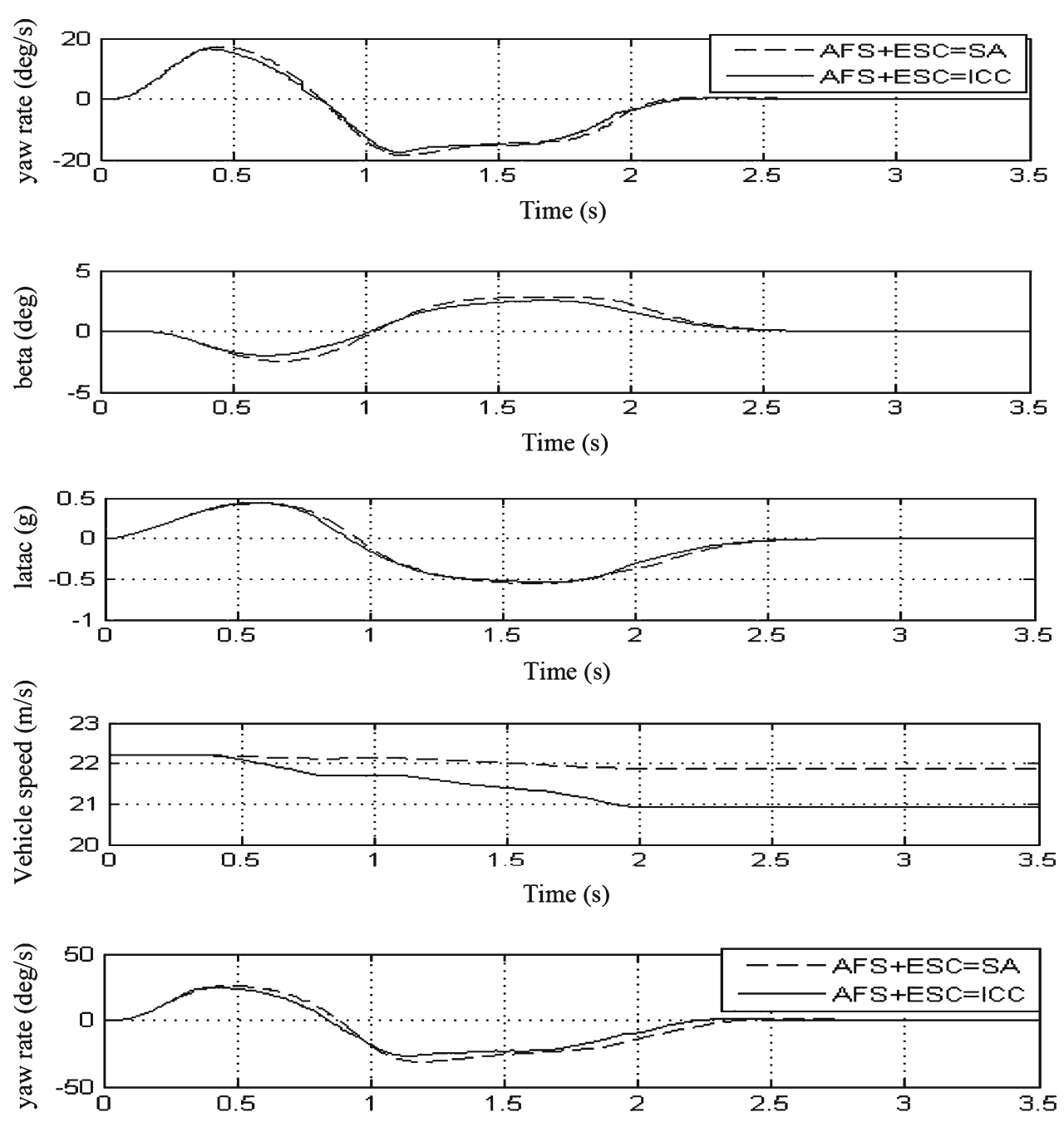

Time (s)
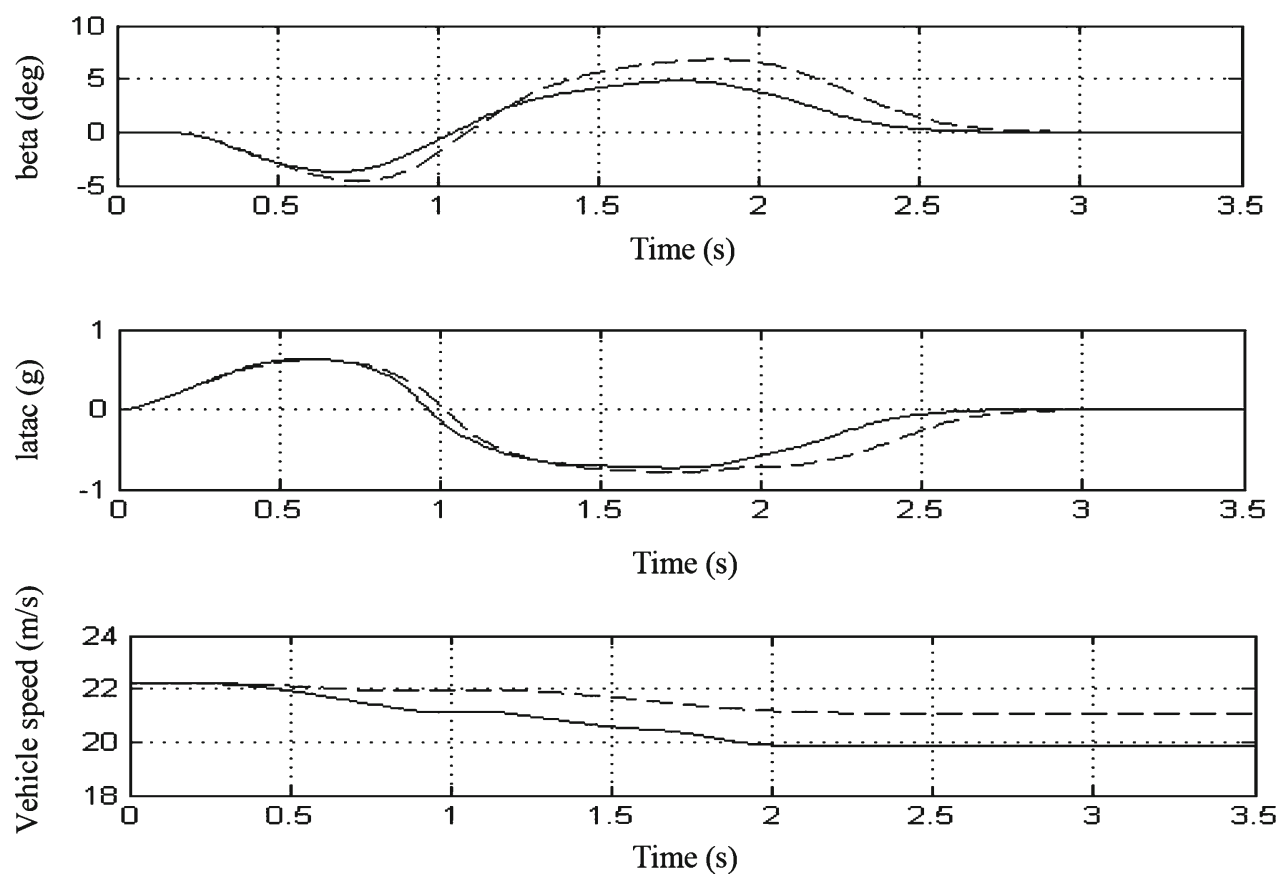
Fig. 29 Schematic of AFS + ESC + VTD standalone controller

Fig. 30 Schematic of AFS + ESC + VTD integrated controller (ICC)
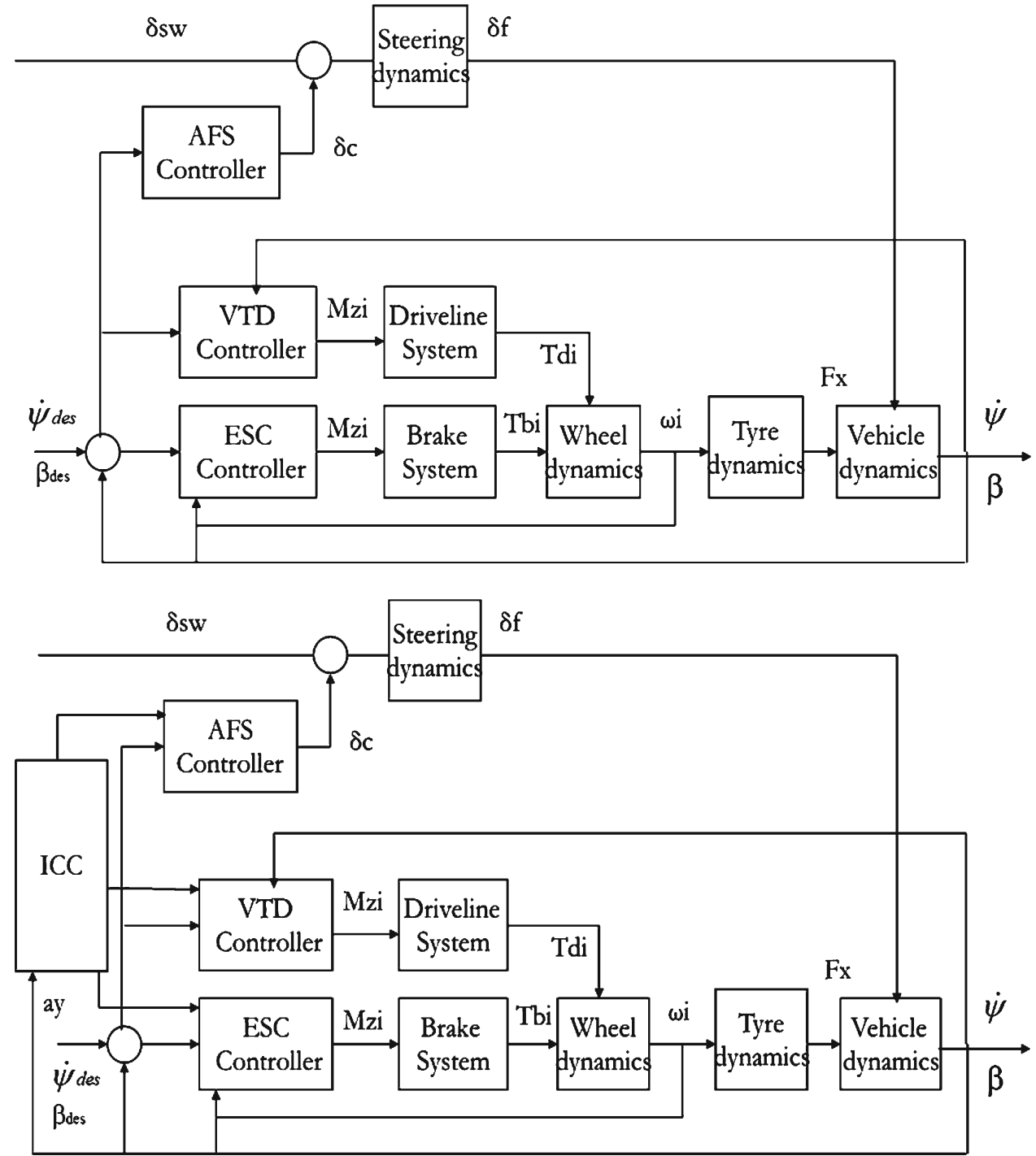

The relationship between the operating point and the lateral acceleration is a function of the road surface coefficient of friction. It is assumed that the road surface coefficient of friction can be measured or estimated. Hence lateral acceleration can be used as a measure of the operating vehicle point in the integration strategy.

\subsection{Integration of ESC and AFS}

Having investigated the individual behaviour and the control authorities of each of the four chassis control systems, the development of integrated control strategy is carried out as follows. First the ESC and the active front steering systems are activated individually and the vehicle yaw rate, sideslip angle, lateral acceleration and the longitudinal vehicle speed are recorded. Then both of these control systems are activated in standalone mode and the results are compared against that of the individual controllers.
From Fig. 24, when AFS and ESC are activated in a standalone manner, they reduce the yaw rate and the sideslip angle better than when they are activated individually. This shows that both the AFS and the ESC controllers complement each other in improving the vehicle handling performance. Compared to the ESC only activated scenario, the AFS and ESC standalone controller performs less intrusive in reducing the longitudinal vehicle speed. But AFS still dominates in providing the less safety critical low latac region of vehicle operation as shown in Fig. 25. Again, both in the medium and high latac regions the AFS + ESC standalone controller performed better than the individual ones.

Following the above analysis of the AFS and ESC in standalone manner on low, medium and high latac regions, a rule based ICC strategy is developed (Fig. 26). The developed integrated controller has one input and two outputs. The vehicle lateral acceleration is fed back to the integrated controller as the input and is used to determine the vehicle operating 
Fig. 31 Performance of ICC $(\mathrm{AFS}+\mathrm{ESC}+\mathrm{VTD})$ at medium latac
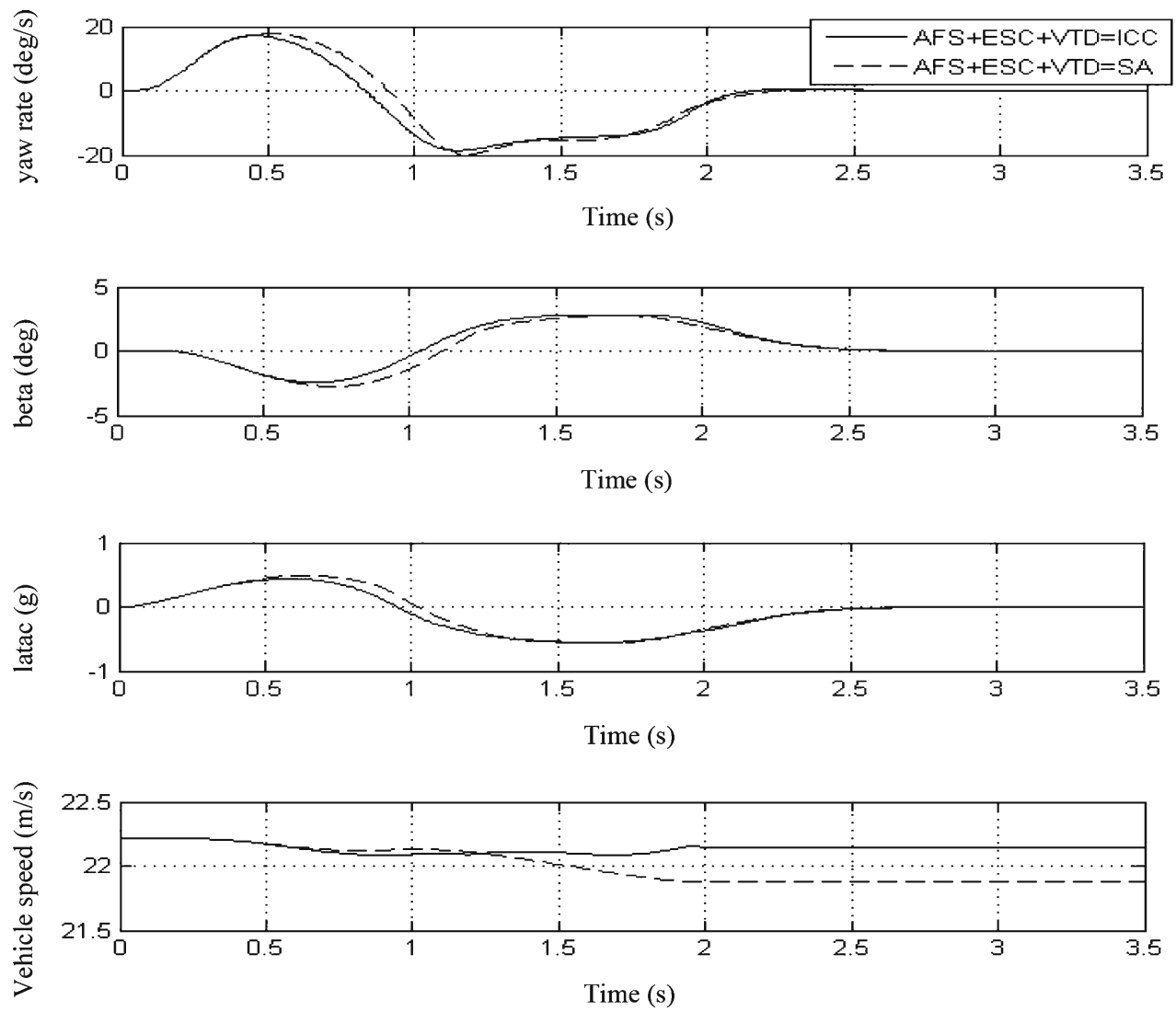

Fig. 32 Performance of ICC $(\mathrm{AFS}+\mathrm{ESC}+\mathrm{VTD})$ at high latac
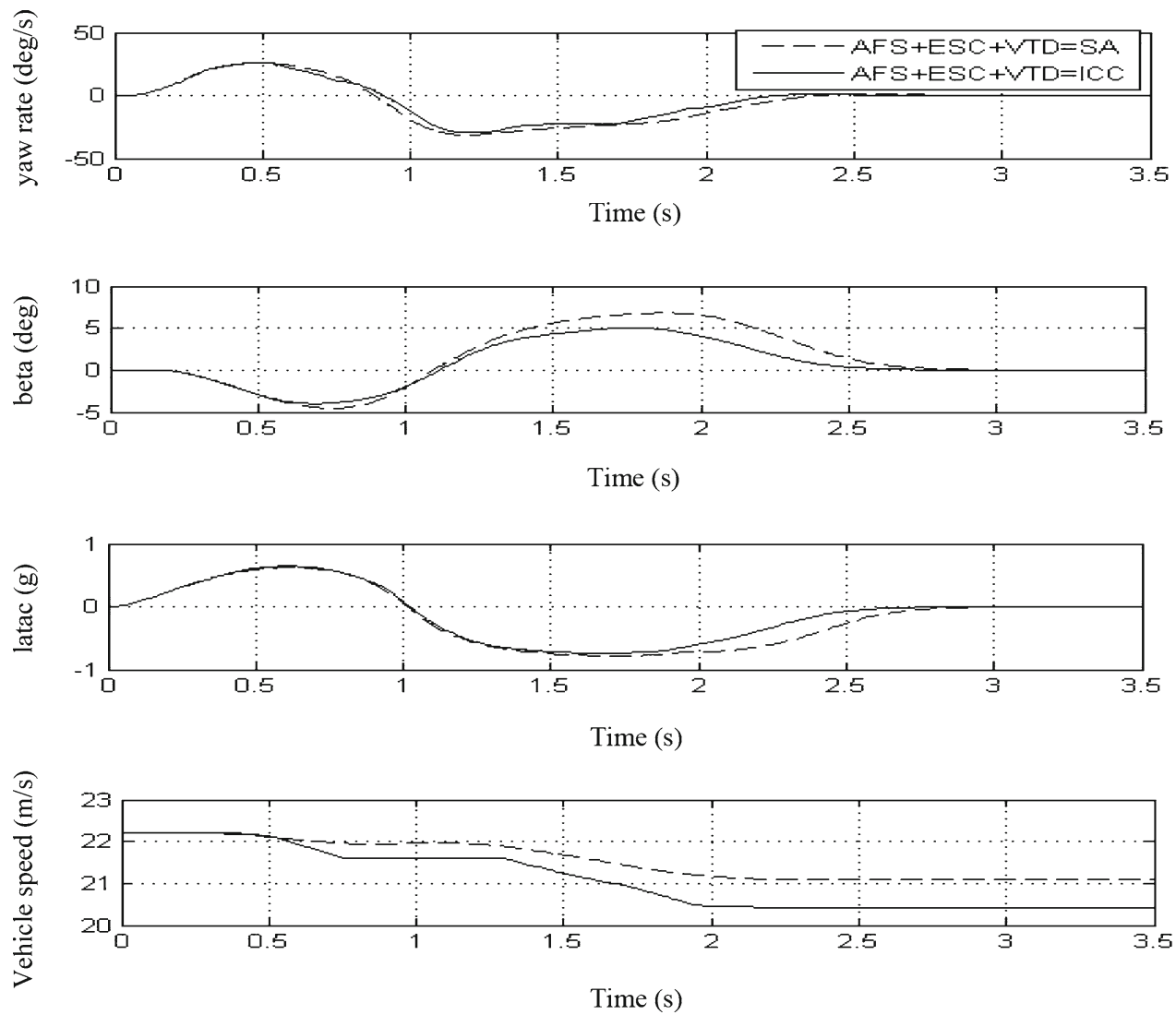
Fig. 33 Schematic of AFS + $\mathrm{ESC}+\mathrm{VTD}+\mathrm{NFC}$ standalone controller

Fig. 34 Schematic of AFS + $\mathrm{ESC}+\mathrm{VTD}+\mathrm{NFC}$ integrated controller (ICC)
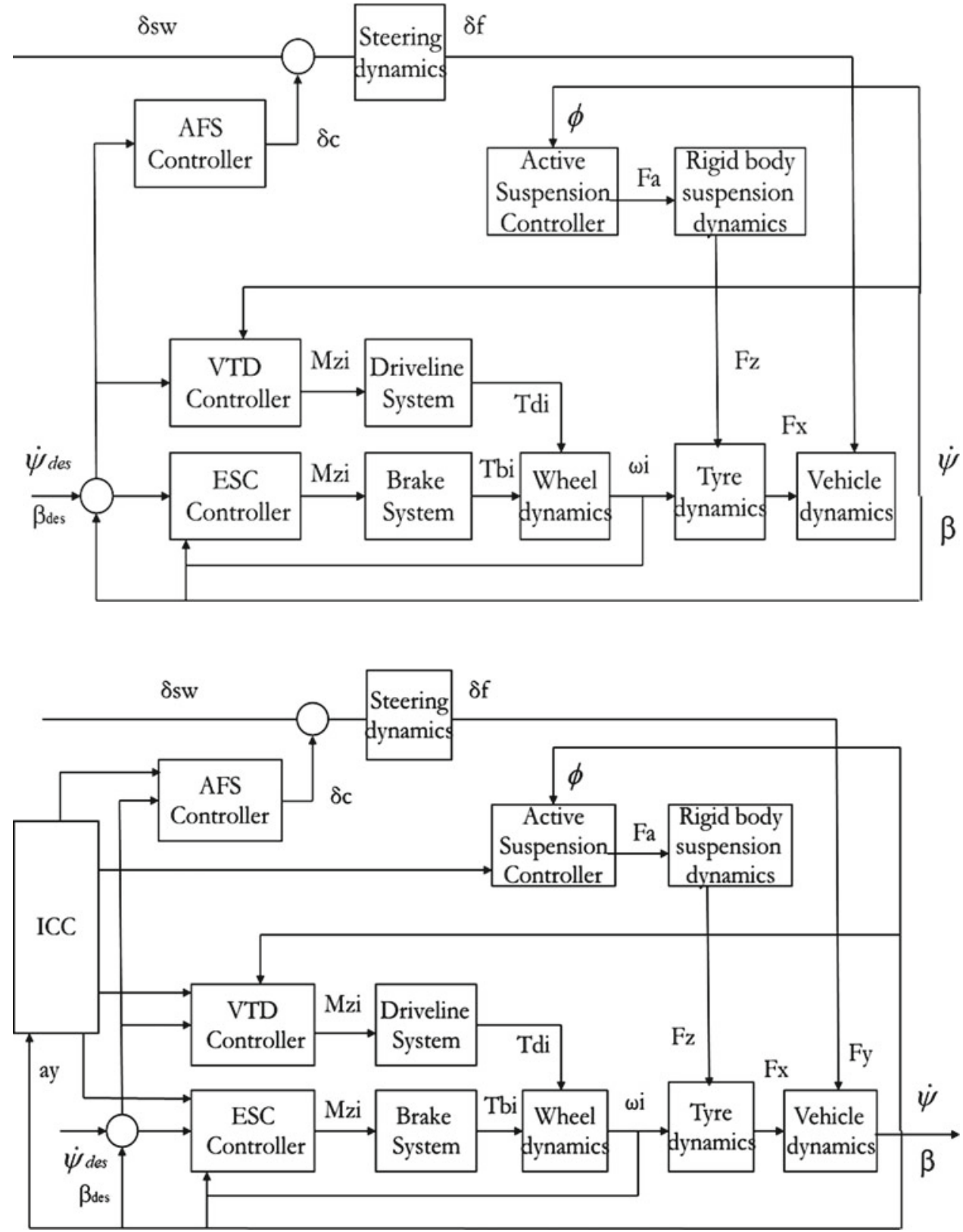

region. Having determined the vehicle operating region, the integrated controller allocates the vehicle dynamics control authority between the AFS and the ESC.

The rule based integrated controller activates the AFS in the low latac range until $0.3 \mathrm{~g}$ and then handover the control authority to ESC. As the low latac range is within $0.3 \mathrm{~g}$, the ICC utilises the ability of the AFS to reduce the vehicle yaw rate and sideslip angle. At the same time, since the ESC is not activated, the ICC does not intrude in the vehicle longitudinal dynamics.

From the Figs. 27 and 28, when the vehicle is operated in the medium and high latac regions, the integrated controller performs better than the standalone controller in improving the vehicle handling. Due to the deactivation of AFS and the intervention of ESC beyond the $0.3 \mathrm{~g}$ latac, the exit speed of the manoeuvre is less than the standalone controller, better than ESC only system.

So in summary, the integrated controller (AFS + ESC) performs at par with the standalone system in the low latac and performs better than standalone controller by reducing the vehicle yaw rate and sideslip angle at the medium and the high latac regions. The exit speed of the manoeuvre with ICC is less than the standalone controller, but, better than the ESC only system. 
Fig. 35 Performance of ICC $(\mathrm{AFS}+\mathrm{ESC}+\mathrm{VTD}+\mathrm{NFC})$ at medium latac
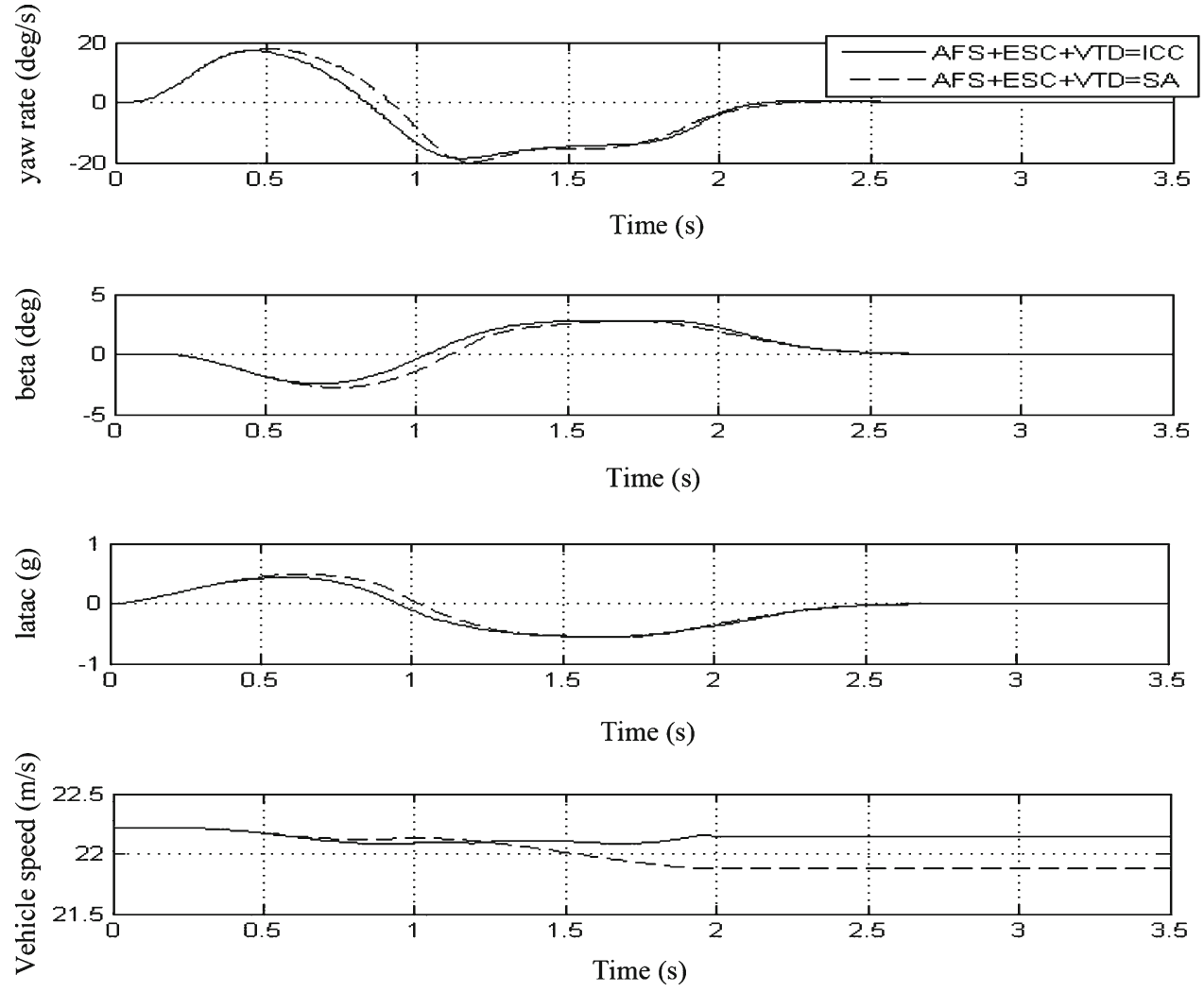

\subsection{Integration of ESC, AFS with VTD}

Having integrated the AFS and the ESC systems, this section investigates the integration of VTD with the integrated controller developed in the previous section. From the standalone controller analysis in the earlier sections, the control authority of the AFS diminishes at the medium and the high latac regions and also less intrusive at the less critical, low latac region. So the further integration strategy deactivates the AFS at the limits of low latac and considers the next two key stability control systems, VTD and ESC. Both VTD and ESC are effective in improving lateral handling of the vehicle at the medium latac zone, but the VTD limits the reduction in vehicle longitudinal speed compare to the more intrusive ESC.

So, the integrated control strategy activates only the AFS at the low latac and the VTD at medium latac. For the high and limit latac the ESC is activated. This integration strategy optimises the use of these three active chassis systems at the same time improves the vehicle handling without reducing the current vehicle performance, such as maintain or negligible effects of longitudinal vehicle speed. Figures 29 and 30 shows the schematics of AFS, VTD and ESC controllers in standalone and integrated modes.

The rule based integrated controller is enhanced to accommodate the necessary extra rules to integrate the VTD system to the existing integrated controller. From the Figs. 31 and 32 , when the vehicle is operated in the medium and high latac regions, the integrated controller performs better than the standalone controller in improving the vehicle handling. Due to the activation of VTD and the deactivation of ESC in the medium latac zone of $0.3-0.6 \mathrm{~g}$, the exit speed of the manoeuvre is better than the AFS + ESC ONLY integrated controller system.

In summary, the integrated controller (AFS + VTD + ESC) performs at par with the (AFS + ESC) integrated control system in the low, medium and high latacs and performs better than the standalone controller across the all latac regions. The exit speed of the manoeuvre with ICC is better in the medium latac range due to the activation of VTD.

\subsection{Integration of ESC, AFS, VTD with NFC}

From the individual chassis controller analysis the NFC controller has little or no effect at the low latac and a moderate effect on improving the vehicle handling in the medium latac region. Its ability to generate the extra tyre forces depends mainly on the amount of lateral and longitudinal load transfer. When NFC is activated, only the steering input is given to the vehicle. Hence the additional normal force on the wheels influenced only the lateral tyre forces. 
Fig. 36 Performance of ICC $(\mathrm{AFS}+\mathrm{ESC}+\mathrm{VTD}+\mathrm{NFC})$ at high latac
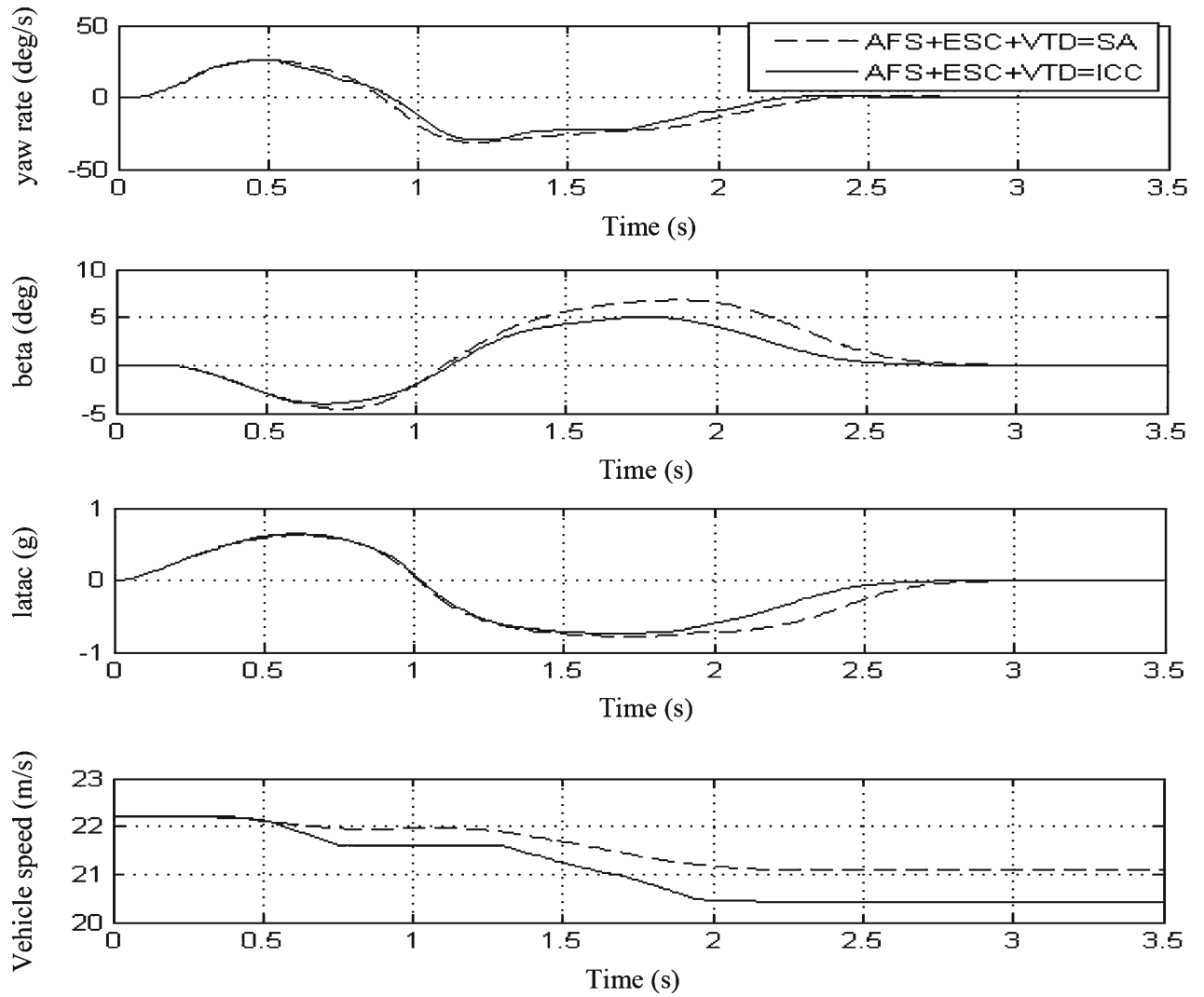

The effect of NFC on the longitudinal forces will add more influence on generating the corrective yaw moment. A schematic diagram of the AFS, ESC, VTD and NFC controllers in standalone manner is given on Fig. 33.

A further enhancement is made to the rule based integrated to accommodate the necessary rules to integrate the NFC system to the existing integrated controller. This fully integrated chassis controller (ICC), integrates the ESC, AFS, VTD and suspension NFC. This rule based ICC strategy provides the control authority to AFS at the low latac range, to VTD at medium latac range, to ESC at high and at limits and activates the NFC from medium latac onwards to optimise the generation of lateral and longitudinal tyre forces and to use the four active chassis systems effectively.

A schematic of the novel four systems ICC control strategy is given in Fig. 34. From the Figs. 35 and 36, when the vehicle is operated in the medium and high latac regions, the integrated controller performs better than the standalone controller in improving the vehicle handling. In summary, the integrated controller (AFS + VTD + ESC + NFC) performs at par with the (AFS + ESC + VTD) integrated control system in the low latac region and performs better in the medium to high latac and at the limits.

\section{Conclusions}

A detailed non-linear vehicle dynamics model is developed and four standalone vehicle control systems AFS, ESC, VTD and NFC are modelled and simulated in MATLAB/Simulink. In some cases, researcher used standard commercially available softwares such as CarSim and MSc. ADAMS as their base vehicle models. Most of the studies reviewed, considered only two standalone chassis systems that represent any two of the four key vehicle functional domains for the purpose of integration. Those approaches either provide little or no interaction between various key DoF of a vehicle and their effects of each other. In this paper, various possible combinations of integrated strategies between these four systems are investigated through simulations. Based on these investigations a new integrated control strategy is proposed to make these four systems cohabit to improve the present vehicle handling performance. Simulations with the integrated controller demonstrated a significant improvement in the performance objectives. The vehicle motion during an emergency maneuver is much improved, showing better handling and characteristics during sudden, high speed maneuvers together with a corresponding reduction in yaw rate and sideslip angle. The significant conclusions here is that the AFS, ESC, VTD and NFC individually have a significant positive 
influence on vehicle handling and integrating these four systems showed that they can be a part of future global chassis control strategies.

\section{References}

1. Anthony B, Zak S (2000) Antilock brake system modelling and fuzzy control. Int J Veh Des 24(1):1-18

2. Beyer C, Dominke, P, Sonntag E (1993) Anti-lock control system. US Patent 5249852

3. Bowman J (1993) Feasibility study of an automotive slip control braking system. SAE Paper 930762

4. Lin J, Ting W (2007) Nonlinear control design of anti-lock braking systems with assistance of active suspension. IET Control Theory Appl 1(1):343-348

5. Cooper N (2005) Integration of active suspension and active driveline to ensure stability while improving vehicle dynamics. SAE Paper 2005-01-0414

6. Rengaraj C (2012) Integration of active chassis control systems for improved vehicle handling performance. $\mathrm{PhD}$ thesis, University of Sunderland, UK

7. Junjie H, Crolla D, Levesley M (2006) Coordination of active steering, driveline and braking for integrated vehicle dynamics control. Proc IMechE D 220:1401-1421
8. Karbalaei R, Ghaffari A, Kazemi R, Tabatabaei S (2007) A New intelligent strategy to integrated control of AFS/DYC based on fuzzy logic. Int J Eng Appl Sci 3(5):264-269

9. Kazuya K, Peng $\mathrm{H}(2000) \mathrm{H}_{\infty}$ control for integrated side-slip, roll and yaw controls for ground vehicles. In: Proceedings of AVEC 2000 , 5th international symposium on advanced vehicle control, Ann Arbor, MI, USA, August 22-24

10. March C, Shim T (2007) Integrated control of suspension and front steering to enhance vehicle handling. Proc IMechE D 221(4):377509

11. Pacejka H, Besselink I (1997) Magic formula tyre model with transient properties. Veh Syst Dyn 27(1):234-249

12. Bakker E, Myborg L, Pacejka H (1987) Tyre modelling for use in vehicle dynamics studies. SAE Paper No. 870421

13. Rajamani R, Hedrick J (1995) Adaptive observers for active automotive suspensions - theory and experiment. IEEE Trans Control Syst Technol 3(1):86-93

14. Fletcher I, Arden W, Cox C (2004) A benchmark for ABS control algorithm evaluation. In: 2004 JSAE annual congress proceedings, Yokohama, Japan, May 19-21

15. Rajamani R (2006) Vehicle dynamics and control. Springer, New York, ISBN 978-0-387-26396-0

16. Pinnel A, Butz T, Ehmann M, Fan H (2004) Vehicle dynamics simulation with active yaw control using variable drive torque distribution. In: 2004 JSAE annual congress proceedings, Yokohama, Japan, May 19-21 